\title{
Disociaciones entre pruebas implícitas y explícitas de memoria: significado e implicaciones teóricas
}

\author{
José María Ruiz-VARGAS \\ Universidad Autónoma de Madrid
}

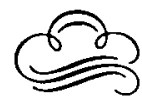

Resumen

Las disociaciones entre pruebas implícitas y explícitas de memoria se ban convertido últimamente en el fenómeno más rico e ilustrativo teóricamente de que la memoria se manifiesta tanto de una forma directa o explícita como de una forma indirecta o implícita. La distinción entre memoria implicita $y$ explícita ba proporcionado un marco teórico y de trabajo extremadamente fructífero para aumentar nuestro conocimiento sobre la naturaleza y arquitectura de la memoria. En este trabajo se presenta una amplia revisión de las disociaciones entre pruebas implícitas y explícitas de memoria, las múltiples variables que las producen, las distintas poblaciones susceptibles de generarlas y las explicaciones más relevantes sobre su significado. De entre estas explicaciones destacan: 1) la que considera que las disociaciones son el resultado de diferentes procesos y 2) la que entiende que reflejan diferentes sistemas de memoria. Entre los proponentes de ambas explicaciones se ba desarrollado un debate que ba llevado a una nueva concepción de la naturaleza y arquitectura del sistema de memoria que da fundamento a los efectos de priming.

Palabras clave: Disociaciones de memoria, memoria implícita y explícita, pruebas implícitas y explícitas, priming, amnesia, sistemas de memoria.

\section{Abstract}

The dissociations between implicit and explicit memory tests bave lately become the most outstanding proof that memory may manifest itself either in a direct, explicit way or in an indirect or implicit way. This implicit versus explicit memory distinction bas provided an extremely fruitful theoretical and empirical framework to gain knowledge about the nature and the architecture of memory. This paper provides a comprebensive review of the dissociations between implicit and explicit memory tests, alongside the many variables that cause them, the various populations that may generate them, and the most relevant accounts of their meaning. The two most influential accounts run as follows: 1) dissociations arise as a consequence of different processes, and 2) they reflect different memory systems. The ensuing debate between these proposals bas led, after a decade or so, to a new view about the nature and the architecture of the memory system which lends support to priming effects.

Key words: Memory dissociations, implicit and explicit memory, implicit and explicit tests, priming, amnesia, memory systems.

Este trabajo forma parte del Proyecto de Investigación PB89-0160 subvencionado por la Dirección General de Investigación Científica y Técnica (DGICYT)

Dirección del autor: Departamento de Psicología Básica. Facultad de Psicología. U.A.M. 28049 - Madrid. 


\section{INTRODUCCION}

Si se hace un seguimiento minucioso de lo que está ocurriendo en los últimos años en el campo de la investigación experimental de la memoria, no deja de sorprender - por muy obvio y evidente que parezca - lo que la acumulación de datos empíricos rigurosos puede hacer avanzar una ciencia o un aspecto concreto de una ciencia. La historia reciente de la psicología cognitiva de la memoria nos muestra que ésta tuvo una época dorada en la década de los sesenta. En aquellos años se produjo una auténtica eclosión de teorías, modelos, taxonomías, paradigmas experimentales, tareas y datos que permitieron conceptualizar, abordar experimentalmente e interpretar coherentemente cómo nuestras experiencias pasadas influyen en nuestra experiencia y conducta presentes. Hacia mediados de los setenta, no obstante, aquel panorama pareció haber tocado techo, e incluso dio la sensación de que se entraba en un proceso de involución: no sólo no se avanzaba, sino que parte del trabajo hecho parecía haber servido para poco (ver Neisser, 1978). En nuestra opinión, lo que le ocurrió a la psicología de la memoria fue lo que le ocurriría a cualquier disciplina experimental si las herramientas metodológicas no cubren un amplio abanico de posibilidades; a saber, que los objetivos de estudio acaban configurando un panorama cada vez más restringido que acarrea, antes o después, una insatisfacción creciente entre los investigadores. En la psicología experimental de la memoria y siempre desde la perspectiva del momento actual-, se podrían identificar dos elementos como responsables de la situación de impasse característica de la segunda mitad de los setenta y primeros ochenta. Estos serían: 1) el tipo de medidas de memoria utilizadas por los investigadores dentro y fuera del laboratorio, que, al estar circunscritas al recuerdo y al reconocimiento, habían consolidado la idea (errónea, a todas luces) de que memorizar supone siempre la recuperación consciente o explícita de la información; y 2) la desconexión entre la psicología cognitiva de la memoria y el área que en los últimos años aparece claramente definida como la neuropsicología cognitiva de la memoria.

Ha sido en la década de los ochenta, cuando se han producido los cambios que han afectado a ambos elementos drásticamente. Lo interesante, aunque posiblemente cargado de lógica, ha sido que estos cambios se han producido de forma encadenada. Para nuestros propósitos, lo que nos importa señalar es que, gracias al trabajo pionero de dos neuropsicólogos de la memoria (Warrington y Weiskrantz, 1968, 1970) que, saliéndose de la corriente oficial de la época, evalúan la memoria de pacientes amnésicos con tareas que no encajaban en ningún formato de las medidas tradicionales de recuerdo y reconocimiento, se inicia, por un lado, una conexión entre psicología y neuropsicología de la memoria, que está resultando cada vez más fructífera, $\mathrm{y}$, por otro, toda una revolución en el modo de medir la influencia de eventos pasados en la conducta y experiencia presentes que ha producido en los últimos años una auténtica proliferación de tareas experimentales de memoria (ver las revisiones de Richardson-Klavehn y Bjork, 1988, y Hintzman, 1990). Este nuevo panorama teórico y metodológico ha dado tal empuje a la investigación experimental de la memoria, que estamos asistiendo a uno de los momentos de avance más espectacular y significativo en nuestra com. prensión de la naturaleza y funciones de la memoria humana.

Hasta finales de los sesenta, aproximadamente, se creía que los pacientes amnésicos se caracterizaban por su incapacidad para realizar nuevos aprendizajes. Se asentó y generalizó la idea de que los amnésicos son incapaces de trans- 
ferir información desde el almacén a corto plazo (que, frecuentemente, se mantiene relativamente intacto) al almacén a largo plazo (e. g., Baddeley y Wa. rrington, 1970). En realidad, algunos investigadores habían sido muy precisos al comprobar que amnésicos profundos, como el paciente $H$. M., podían aprender y retener habilidades motoras casi tan bien como los sujetos normales, aunque su capacidad para retener la información verbal estaba claramente dañada (e. g., Corkin, 1968). Los trabajos de Warrington y Weiskrantz $(1968,1970)$, en los que nos detendremos más adelante, presentaron argumentos sólidos para empezar a cambiar las ideas más fuertemente establecidas acerca de la incapacidad de los amnésicos para adquirir información nueva. Estos investigadores demostraron que los pacientes amnésicos aprendían material verbal al mismo nivel que los sujetos de control si, en lugar de medir su retención con pruebas estándar de reconocimiento o recuerdo, se les administraban pruebas que evaluaban indirecta o implícitamente el aprendizaje.

En los estudios pioneros de Warrington y Weiskrantz $(1968,1970)$ encontramos los primeros usos de lo que hoy llamamos pruebas implícitas de memoria, así como evidencia experimental de disociaciones entre pruebas implícitas y explícitas de memoria, lo que parece indicar que existe una diferencia fundamental entre la información mnemónica medida con tests implícitos y la medida con tests explícitos de memoria.

\subsection{Pruebas implícitas y explícitas de memoria}

La distinción entre pruebas o tests implícitos y explícitos de memoria está estrechamente relacionada con la distinción entre memoria implícita y explícita. Aunque hay evidencia histórica del uso de tal distinción desde mediados del siglo XVII (ver Schacter, 1987, para una revisión), el término «memoria implícita» no se introduce hasta 1985. Fueron Graf y Schacter (1985) los que definieron, por primera vez, los términos «memoria implícita» y «memoria explícita». La memoria implícita es aquella que se revela «cuando la ejecución de una tarea se ve facilitada en ausencia de recolección consciente» (Graf y Schacter, 1985, p. 501). Esto significa que la memoria implícita se refiere a la retención de información adquirida durante un episodio específico previo y se manifiesta en pruebas en las que a los sujetos no se les pide que recuperen deliberada o conscientemente la información estudiada o el propio espisodio de estudio (Graf y Schacter, 1985; Schacter, 1987, 1990b). Por otra parte, la memoria explícita es aquella que se manifiesta «cuando la ejecución de una tarea requiere la recolección consciente de experiencias previas» (Graf y Schacter, 1985, p. 501). Esta última forma de memoria se refiere, pues, a la recolección consciente o deliberada de información o episodios previos, tal y como se pone de manifiesto en los tests tradicionales de recuerdo libre, recuerdo con claves y reconocimiento.

Parece razonable, entonces, considerar que las pruebas explícitas (e. g., recuerdo y reconocimiento) son medidas directas de memoria, y que las pruebas implícitas (e. g., tareas de decisión léxica y de identificación perceptiva) son medidas indirectas.

Conviene, no obstante, hacer algunas matizaciones acerca de esta terminología dado que en la actualidad existe un cierto desacuerdo al respecto. Nos referimos al hecho de que no todos los autores consideran adecuado establecer una equivalencia total y, por tanto, la posibilidad de intercambio entre los términos «directo» y «explícito», por un lado, e «indirecto» e «implícito», por otro. 


\section{4}

Por ejemplo, Schacter (1987) utiliza la distinción «memoria implícita y explícita» para referirse tanto a las pruebas de memoria como a las formas de memoria (pero no para referirse a sistemas diferentes de memoria); mientras que RicharsonKlavehn y Bjork (1988) plantean que es preferible utilizar la distinción «directo/indirecto" para describir las dos clases de pruebas (como hacen Johnson y Hasher, 1987) y reservar los términos «implícito/explícito» para distinguir formas de memoria (una propuesta idéntica se encuentra en Merikle y Reingold, 1991). Entre las distintas razones aducidas por Richardson-Klavehn y Bjork (1988), resulta especialmente relevante tener en cuenta que, en efecto, no siempre hay seguridad de que un test implícito se ejecute basándose exclusivamente en la memoria implícita. Estos autores se refieren, concretamente, al hecho de que un test nominalmente implícito puede no ser siempre un test funcionalmente implícito. Sin embargo, estamos completamente de acuerdo con Schacter (1990b) cuando responde a tal propuesta señalando que se puede hacer la misma observación sobre la distinción directo/indirecto: durante la realización de un test nominalmente «indirecto» como, por ejemplo, completar palabras fragmentadas, aunque a los sujetos no se les exija que piensen en el episodio de estudio, cabe siempre la posibilidad de que algunos descubran la naturaleza de la tarea y adopten estrategias de recuperación intencionales y explícitas, y, consecuentemente, transformen el test «indirecto» en un test «directo». Schacter, Bowers y Booker (1989) han propuesto para evitar tales situaciones un «criterio de intencionalidad de recuperación» basándose en datos empíricos. Sin embargo, la cuestión clave para cualquiera de las dos terminologías (explícito/implícito y directo/indirecto) es que - como señala Schacter (1990b) - la caracterización nominal de la tarea no puede describir siempre con exactitud el tratamiento real que le dará el sujeto. Por ello, este autor considera más simple y menos problemático utilizar los términos implícito/explícito para referirse tanto a los tests como a las formas de memoria.

No quisiéramos alargarnos más con esta cuestión, aunque sí queremos dejar constancia de que Roediger (1990b) es igualmente partidario de la distinción implícito/explícito. De las razones teóricas y pragmáticas que esgrime en favor de su postura, nos parece especialmente relevante la observación de que la distinción implícito/explícito refleja mejor la idea de diferentes «modos de recuperación» (Roediger y Blaxton, 1987a) o diferentes formas de memoria, al tiempo que implica que el acceso a la memoria se lleva a cabo de un modo diferente en cada caso. La distinción directo/indirecto parece significar que se está accediendo al mismo conocimiento o forma de memoria pero directamente en un test e indirectamente en el otro. Si fuese así, continúa argumentando Roediger (1990b), no cabría esperar las claras disociaciones que frecuentemente se producen entre los tests implícitos y explícitos de memoria. En consonancia con los argumentos utilizados por Schacter y Roediger, nosotros también consideramos que sería aconsejable utilizar (y aquí la utilizaremos) la distinción implícito/explícito para referirse tanto a las tareas como a las formas de memoria.

\subsection{Las disociaciones y su lógica}

Warrington y Weiskrantz $(1968,1970)$ observaron que cuando medían el nivel de aprendizaje de los pacientes amnésicos con tests implícitos y explícitos, se producía una fuerte disociación entre ambos tipos de tests: mientras que la ejecución era, como se esperaba, extremadamente mala en tests de recuerdo y 
reconocimiento, los mismos pacientes amnésicos tenían una ejecución normal en tareas que no requerían la recolección consciente del episodio de aprendizaje. En uno de aquellos estudios, Warrington y Weiskrantz (1970, experimento 2) evaluaron la retención de cuatro pacientes amnésicos (uno con una lobotomía temporal y tres con psicosis alcohólica de Korsakoff) y ocho pacientes de control (con enfermedad neurológica extracerebral). La tarea de aprendizaje consistió en leer en voz alta una lista de ocho palabras tres veces. La retención fue evaluada con cuatro tests diferentes: dos de memoria explícita (recuerdo y reconocimiento) y dos de memoria implícita (identificación perceptiva de palabras degradadas y completar palabras a partir de las tres primeras letras). Los resultados permitieron observar que los pacientes amnésicos habían tenido un rendimiento significativamente peor que los controles en los tests de recuerdo y reconocimiento (pruebas explícitas), y, sin embargo, y he aquí el hallazgo más interesante, habían identificado y completado las palabras (pruebas implícitas) tan bien como los sujetos de control. En resumen, Warrington y Weiskrantz habían encontrado una fuerte disociación entre las hoy llamadas pruebas explí. citas e implícitas de memoria.

Resultados similares a los de Warrington y Weiskrantz (1970) han sido encontrados, muchas veces desde entonces, con otras poblaciones de pacientes, con sujetos normales y con una diversidad de tareas (e. g., Jacoby y Witherspoon, 1982; Tulving, Schacter y Stark, 1982; Jacoby, 1983; Graf, Squire y Mandler, 1984; Graf y Schacter, 1985; Light et al., 1986; Schacter y Graf, 1986a, b; Roediger y Blaxton, 1987a; Hayman y Tulving, 1989a, b; Shimamura y Squire, 1989; Heindel et al., 1989; Butters et al., 1990; Schacter et al., 1990a, b; Schacter et al., 1991; Schacter et al., 1990; ver, además, las revisiones de Schacter, 1987; Richardson-Klavehn y Bjork, 1988, y Roediger et al., 1989). Es precisamente el hecho de que las disociaciones se produzcan en una variedad de situaciones experimentales y con poblaciones muy distintas, lo que confiere al fenómeno un gran interés teórico.

Como señaló Tulving (1983), la lógica de las disociaciones implica que se manipule una sola variable y que sus efectos sean observados y comparados en dos tareas diferentes. Si se comprueba que la variable manipulada ha afectado a la ejecución de los sujetos en una tarea pero no en la otra o que ha afectado a la ejecución en ambas tareas pero en direcciones diferentes, entonces se dice que se ha producido una disociación experimental. En realidad, el tipo de disociación referido es llamado por Tulving y Schacter (1990) disociación funcional. Según estos autores, si la variable independiente es una variable de sujetos, por ejemplo, normales versus amnésicos o ancianos versus jóvenes, los efectos distintos que se produzcan en los dos tests se dice que son debidos a una disociación neuropsicológica o evolutiva, respectivamente. Tulving y Schacter distinguen además un tercer tipo de disociación, que es la que se puede observar cuando se compara una sola población de sujetos en dos tests sucesivos con el mismo conjunto de ítems. Si los resultados de las dos pruebas no correlacionan, se dice que se ha producido una disociación contingente, o una independencia estocástica, entre los tests.

En términos generales, las disociaciones entre pruebas implícitas y explícitas parecen indicar que ambos tipos de pruebas activan o implican formas diferentes de retención. En concreto, las disociaciones están justificando el supuesto de que las pruebas de memoria implícita involucran una forma de retención inconsciente, mientras que las pruebas de memoria explícita implican una forma 
de retención consciente (Roediger, 1990a). El presupuesto teórico del que se parte se ajusta a la lógica siguiente: si las dos pruebas que se comparan representan las operaciones de un solo conjunto o de un conjunto altamente correlacionado de procesos, entonces la comparación de dichas pruebas no debería producir disociación alguna; pero si tal comparación revela una disociación, entonces los procesos implicados tienen que ser diferentes (Tulving y Schacter, 1990).

\subsection{Priming, tareas de priming y relaciones entre priming y memoria explícita}

En los tests implícitos, la medida de interés es el priming o la facilitación en el procesamiento de un estímulo como una función de la presentación previa y reciente del mismo estímulo o de un asociado (Cofer, 1967). La ejecución normal que Warrington y Weiskrantz (1970) observaron en los pacientes amnésicos cuando ejecutaban las tareas de identificar y completar palabras es explicada actualmente en términos de «efectos de priming». El aspecto verdaderamente sorprendente de este hallazgo es que los pacientes amnésicos mostraban una escasa o nula memoria explícita del episodio en el que habían adquirido el conocimiento. En realidad, los pacientes amnésicos de Warrington y Weiskrantz no recordaban que se les hubiera presentado lista alguna de palabras y se enfrentaban con los tests de identificar y completar palabras como si se tratase de un «juego de adivinanzas». Como han señalado distintos autores (e. g., Graf y Schacter, 1985; Schacter, 1987), los efectos de priming no necesitan ni implican una recolección consciente o explícita de un episodio previo y, por eso, se dice que reflejan memoria implícita de información estudiada previamente.

El priming ha sido evaluado con una amplia variedad de tareas de memoria implícita. Una prueba se considera que es sensible a los efectos de priming si se demuestra que su ejecución está sesgada por material presentado previamente. Un tipo común de «test de priming» o implícito consiste en completar raíces o fragmentos de palabras con «la primera palabra que venga a la cabeza» (e. g., Warrington y Weiskrantz, 1968, 1970; Tulving, Schacter y Stark, 1982; Graf y Mandler, 1984; Schacter y Graf, 1986a, b; Roediger y Blaxton, 1987b). Los efectos de priming han sido evaluados también con tareas implícitas que requieren la identificación (perceptiva) de palabras expuestas brevemente (e. g., Jacoby, 1983; Light y Singh, 1987) o la identificación de objetos a partir de la exposición breve de dibujos (e. g., Weldon y Roediger, 1987; Mitchell y Brown, 1988), así como con tareas de decisión léxica (e. g., Scarborough, Gerard y Cortese, 1979; Kirsner, Milech y Standen, 1983).

Una cuestión fundamental que conviene tener presente con respecto a la diversidad de tareas implícitas es que, generalmente, difieren entre sí en cuanto a sus demandas o requerimientos. Llamamos la atención sobre ello porque, como veremos más adelante, este podría ser uno de los factores clave que estaría en la base del desacuerdo teórico existente acerca del significado de las disociaciones entre tests implícitos y explícitos de memoria. Al margen de estas diferencias, se dice que se produce priming cuando se incrementa la probabilidad de identificar ítems presentados previamente, o cuando disminuye la latencia de la respuesta de identificación, con respecto a medidas similares ante ítems no presentados. En estos casos, se comprueba que la magnitud del priming aparece indicada por el tamaño de la diferencia entre la exactitud o la latencia de respuesta ante los ítems estudiados y los no estudiados (Schacter, 1990a).

Dado que el resultado más sorprendente de los abundantes estudios con priming es la clara y fuerte disociación que se produce entre memoria implícita 
y explícita, parece pertinente reconocer que otra cuestión de especial trascendencia debe de ser la relativa a las relaciones entre priming y memoria explícita. Según algunos teóricos, existen argumentos para pensar que el priming opera independientemente y no afecta al recuerdo consciente o explícito (Tulving et al., 1982). Según otros, los datos apuntan a que el priming facilita o contribuye al recuerdos explícito (Mandler, 1980; Jacoby, 1983).

La evidencia disponible en uno y otro sentido proviene de estudios con sujetos normales, por un lado, y de estudios con sujetos amnésicos, por otro. Los estudios con sujetos normales han seguido una de las dos siguientes estrategias: a) demostrar que ciertas variables independientes afectan a un test pero no al otro; o b) demostrar la existencia de independencia estocástica entre tests sucesivos. En todos ellos se han tratado de identificar disociaciones entre los tests de priming y los de memoria explícita. Los estudios más relevantes con una y otra estrategia serán descritos en los siguientes apartados cuyo objetivo será analizar en profundidad las múltiples y diversas disociaciones entre priming y memoria explícita. En consecuencia, no nos detendremos ahora en ellos. Sí adelantaremos que esta es la cuestión más conflictiva dentro del amplio debate que en los últimos años se viene manteniendo acerca de la significación teórica de las disociaciones entre tests implícitos y explícitos de memoria. Como veremos, el enfrentamiento entre los que apelan a diferentes procesos y los que postulan la existencia de sistemas diferentes de memoria para explicar las disociaciones entre memoria implícita y memoria explícita va a estar fundamentalmente motivado por la falta de claridad acerca de las bases teóricas subyacentes al fenómeno de priming. Será necesario, por tanto, aclarar al máximo la naturaleza del priming.

En cuanto a los estudios con pacientes amnésicos, la evidencia neuropsicológica aportada parece corroborar la idea de que el priming y la memoria explícita operan independientemente. Squire, Shimamura y Graf (1985) estudiaron la relación entre priming y memoria explícita con pacientes amnésicos sometidos a terapia electroconvulsiva (TEC) para tratarlos de cuadros depresivos. Pruebas piloto habían mostrado que los pacientes de TEC presentaban niveles de reconocimiento cercanos al azar cuando se les evaluaba dentro de la primera hora subsiguiente al tratamiento. Squire et al. utilizaron tests de reconocimiento de elección forzosa con tres alternativas tras cuatro intervalos diferentes (45 minutos, 65 min., 85 min. y 9 horas) tras el tratamiento de TEC. Asimismo, utilizaron tests de completar palabras tras intervalos de 45,65 y 85 minutos. El material consistía en 10 palabras y su retención era medida siempre 15 minutos después de la fase de estudio. En la condición 45 minutos de intervalo tras el tratamiento de TEC, el rendimiento en el test de reconocimiento se situó cerca del nivel de azar ( 3.8 palabras reconocidas; azar $=3.3$ palabras); sin embargo, la ejecución en el test de completar palabras fue equivalente a la de los sujetos de control (pacientes amnésicos $=26 \%$ correcto frente a sujetos de control $=25 \%$ ). Squire et al. (1985) observaron, además, que, a medida que aumenta el intervalo, la ejecución de los pacientes en memoria de reconocimiento iba mejorando sensiblemente, mientras que la ejecución en el test de priming se mantenía estable. Estos hallazgos pusieron de manifiesto la independencia entre la memoria explícita (medida con tests de reconocimiento) y el priming (medido con un test de completar palabras). Así pues, parece que en los pacientes amnésicos el priming no juega un papel significativo en la memoria de reconocimiento.

Ahora bien, existe también evidencia que no encaja aparentemente con es- 
tos resultados. Por ejemplo, Shimamura y Squire (1984) demostraron que el aprendizaje de pares asociados está relacionado con los efectos de priming semántico. En efecto, estos investigadores comprobaron que, en pruebas inmediatas, pacientes con síndrome de Korsakoff mostraban una buena memoria de pares de palabras altamente relacionadas (e. g., MESA-SILLA). En concrecto, Shimamura y Squire observaron que la probabilidad (con respecto a la línea base) de dar la respuesta SILLA ante el estímulo MESA, en un test de asociación libre, era más del doble de la observada con los mismos materiales en un test de recuerdo con claves, en el que las claves eran las palabras estímulo del test de asociación libre. Estos resultados han sido interpretados en términos de efectos de priming de representaciones integradas en memoria semántica. Es decir, la buena memoria de pares asociados altamente relacionados exhibida por los pacientes amnésicos es una función de la capacidad de priming semántico, que se encuentra preservada en estos pacientes. Volveremos sobre este tema más adelante.

Aquí tenemos dos trabajos que parecen ofrecer resultados distintos sobre las relaciones entre priming y memoria explícita. Sin embargo, estos estudios son dos buenos ejemplos para entrever que en muchas ocasiones se están comparando fenómenos distintos desde el presupuesto (equivocado) de su equivalencia. En ambos estudios se habla de priming como de un fenómeno único sin caer en la cuenta de que las demandas de las tareas implícitas correspondientes tienen poco en común. En el estudio de Squire et al. (1985) el test de priming es una prueba perceptual de completar palabras, mientras que en el de Shimamura y Squire (1984) el test de priming es una prueba de asociación libre de pares altamente asociados en memoria semántica. Parece, pues, que sería razonable pensar que estos dos tests implícitos de priming están involucrados a sistemas representacionales distintos. ¿No se hace necesario, entonces, distinguir entre posibles tipos de priming?

\subsection{Priming perceptivo versus priming conceptual}

La cuestión relativa a la necesidad (teóricamente consistente) de distinguir diferentes tipos de priming ha llevado a Tulving y Schacter (1990) a postular explícitamente la distinción entre a) un priming que se expresa en pruebas implícitas en las que el procesamiento está determinado por las propiedades físicas de las claves del test, y b) un priming que se manifiesta en pruebas implícitas en las que se requiere un procesamiento semántico. El primero sería un priming perceptual y el segundo un priming conceptual.

El priming perceptual puede considerarse, como veremos en los apartados siguientes, como un fenómeno presemántico en el sentido de que (1) se produce tanto si los sujetos llevan a cabo operaciones semánticas como si no, y (2) es muy sensible a los cambios que la información pueda sufrir en sus propiedades perceptuales entre las fases de estudio y test. El priming conceptual, por el contrario, puede verse afectado por las operaciones de codificación semántica y, además, es apenas sensible a los cambios de las propiedades superficiales de la información (Schacter, 1990a). El priming perceptual sería equivalente al priming directo o de repetición; el priming conceptual equivaldría al priming indirecto (semántico o asociativo).

Estas distinciones teóricas, avaladas por abundantes datos empíricos, están resultando de una especial utilidad para esclarecer la no escasa cofusión existente acerca de las demandas de las múltiples tareas implícitas de memoria. To- 
do lo que resta de nuestra exposición, así como la discusión final sobre los mecanismos subyacentes a las disociaciones entre tests implícitos y explícitos de memoria o, lo que es lo mismo, sobre el debate «procesos diferentes» versus «sistemas diferentes de memoria», tendrá permanentemente presente esta distinción entre priming perceptual y priming conceptual. En el apartado siguiente, analizaremos con detalle las disociaciones entre priming perceptual y tests explícitos de memoria.

\section{DISOCIACIONES ENTRE PRIMING PERCEPTUAL Y MEMORIA EXPLICITA}

El interés por el estudio de las disociaciones entre priming perceptual y memoria explícita tiene su origen, como ya ha sido apuntado, en los trabajos con pacientes amnésicos de Warrington y Weisktantz $(1968,1970)$. Sus experimentos dejaron claro que los pacientes amnésicos demuestran una buena retención cuando ésta se evalúa con tests de completar palabras presentadas en un episodio anterior, a pesar de su incapacidad para relacionar tales tareas con el episodio en el que se presentaron tales palabras. En los años ochenta, distintos investigadores comprobaron que estas disociaciones también podían producirse en sujetos normales mediante la manipulación de variables experimentales apropiadas (e. g., Jacoby y Dallas, 1981; Graf, Mandler y Haden, 1982; Tulving et al., 1982; Jacoby, 1983). Con estos estudios se inició una línea de investigación que compara sistemáticamente la ejecución en pruebas implícitas y explícitas de memoria. Los abundantes datos generados indican que los efectos de priming perceptual o de repetición pueden disociarse experimentalmente de los de la memoria explícita.

En este apartado se presenta evidencia experimental de numerosas disociaciones que han sido agrupadas en cinco grupos que difieren en cuanto a las poblaciones de sujetos, el tipo de procesamiento de las tareas, los tests, el tipo de claves de recuperación y los tipos de material empleados.

\subsection{Disociaciones entre priming y pruebas explícitas en pacientes amnésicos}

La amnesia está asociada a una disfunción cerebral en la que están involucradas la región temporal medial y las estructuras diencefálicas (Squire, 1987; Weiskrantz, 1987; Mayes, 1988). El síndrome amnésico se caracteriza por un trastorno selectivo de memoria que se produce en ausencia de cualquier otra disfunción perceptiva, lingüística o intelectual (Squire, 1987; Shimamura, 1989). Uno de los aspectos más sorprendentes del síndrome amnésico es el hallazgo de que estos pacientes pueden tener un rendimiento normal o casi normal en una variedad de pruebas implícitas de memoria a pesar de su incapacidad para recuperar el episodio de aprendizaje que constituyó la base de su buena ejecución en tales pruebas. Dicha incapacidad se manifiesta con pruebas explícitas de laboratorio como el recuerdo libre, el recuerdo con claves y el reconocimiento.

Los estudios sobre los efectos de priming en la amnesia fueron inciados por Warrington y Weiskrantz (1968). Desde entonces, se han replicado sistemáticamente sus hallazgos y parece estar sólidamente confirmado el principio de que su buena o mala retención depende esencialmente de la naturaleza implícita o explícita de la prueba. En este sentido, Graf, Squire y Mandler (1984) demostraron que el determinante crítico de la ejecución de los amnésicos era la natu- 
raleza (implícita/explícita) de las instrucciones del test. Estos investigadores presentaron a sujetos amnésicos y de control palabras que tenían que ser analizadas en dos tareas distintas: 1) Una tarea semántica, que consistía en evaluar cada palabra en base a lo agradable o desagradable que resultaba, y 2) una tarea no semántica, que requería determinar si las vocales de la palabra presente eran las mismas que las de la palabra anterior. Como las palabras se habían presentado en una situación de aprendizaje incidental, los sujetos no esperaban ninguna prueba de memoria. A continuación, los sujetos recibieron fragmentos con las tres primeras letras de cada una de las palabras de la fase de estudio y les dieron las instrucciones; o bien explícitas: utilice estos fragmentos como claves para que le sea más fácil recordar las palabras que se le presentaron anteriormente; o bien instrucciones implícitas: complete estas raíces con la primera palabra que le venga a la cabeza. Los resultados no pudieron ser más claros: con instrucciones explícitas, el rendimiento de los amnésicos apareció alterado con respecto a los controles; mientras que con instrucciones implícitas, los amnésicos y los controles mostraron magnitudes comparables de priming. Graf et al. (1984) comprobaron, además, que los efectos de priming no habían sido afectados por el tipo de tarea (semántica versus no semántica).

Las relaciones sorprendentes entre memoria y conciencia de los amnésicos puestas de manifiesto en este estudio no serán tratadas por nosotros en este trabajo (el lector interesado puede consultar los trabajos de Schacter, 1989, 1990c, y Froufe, 1991). Para nuestros propósitos sí resulta relevante destacar del estudio de Graf et al. (1984) la demostración de priming preservado en los pacientes amnésicos. Otras demostraciones han sido ofrecidas por Moscovitch (1982), Cermak et. al. (1985) y Schacter (1985). Shimamura (1986) ha revisado ampliamente la literatura sobre priming preservado en la amnesia y ha encontrado ocho tipos de tests implícitos en los que los pacientes amnésicos han mostrado un efecto priming comparable al de los sujetos de control. Las tareas en cuestión son: completar dibujos fragmentados, completar palabras, decisión léxica, identificación perceptiva, deletrear homófonos, juicios de preferencias, asociación libre de información relacionada y completar palabras con asociados nuevos (para más detalles, ver Shimamura, 1986).

En resumen, la abundancia de estudios experimentales en los que se ha encontrado un priming intacto en pacientes amnésicos, junto a una ejecución deficiente en tareas explícitas, constituye una prueba inequívoca de disociaciones entre priming de repetición y memoria explícita en la amnesia.

\subsection{Disociaciones evolutivas}

El interés por estudiar las disociaciones entre memoria implícita y explícita se ha propagado también al estudio del status relativo de ambas expresiones muemónicas durante el curso del desarrollo de la memoria. Aunque esta línea de investigación se ha iniciado en la segunda mitad de la década de los ochenta, existen ya datos consistentes acerca de la evolución de la memoria implícita y explícita a lo largo de la vida.

Teniendo en cuenta los datos sobre la expresión diferencial de la memoria implícita y explícita en pacientes amnésicos, un grupo de investigadores se planteó la cuestión de si los adultos viejos, con fallos de memoria en su vida contidiana, mostrarían una memoria alterada cuando se les evaluase con pruebas explícitas y una memoria normal cuando fuesen evaluados con pruebas implícitas (e. g., Light, Singh y Capps, 1986; Light y Singh, 1987; Light, 1988; Howard, 1988; 
Java y Gardiner, 1991). Al mismo tiempo, otro grupo se marcó como objetivo estudiar cómo evolucionan ambas memorias durante la niñez (e. g., Schacter y Moscovitch, 1984; Carroll, Byrne y Kirsner, 1985; Parkin y Streete, 1988; Greenbaum y Graf, 1989). De ambas líneas de investigación ha emergido el siguiente cuadro de la evolución de la memoria implícita y explícita a lo largo de la vida: 1) la evidencia parece ser muy clara en cuanto a que la capacidad para la recolección explícita se adquiere y desarrolla durante el período preescolar y escolar, se mantiene relativamente estable durante la vida adulta y declina en la vejez; y 2) los datos parecen apoyar cada vez más la idea de que algunos aspectos de la memoria implícita están ya en funcionamiento al comienzo del período preescolar y se mantienen intactos durante el resto de la vida adulta y la vejez (ver las revisiones de Graf, 1990, y Light, 1991). De todo ello se desprende que las alteraciones de la memoria debidas a la edad están claramente asociadas a la memoria explícita, y que los efectos de priming observados en pruebas de memoria implícita se encuentran relativamente intactos en la vejez.

En una revisión recientísima, Light (1991) presenta una relación de tests implícitos en los que se ha observado el mantenimiento de los niveles de priming a lo largo de toda la vida (ver Tabla I). En los mismos estudios citados, se comprobó, además, que la memoria explícita se expresaba diferencialmente en función del proceso natural de envejecimiento.

TABLA I

Tareas implícitas en las que se ban obtenido niveles similares de priming a lo largo de la vida (Light, 1991)

\begin{tabular}{ll}
\hline \multicolumn{1}{c}{ Tarea } & \multicolumn{1}{c}{ Estudio } \\
\hline 1. Completar fragmentos & Light, Singh y Capps (1986) \\
2. Completar raíces & Howard (1988), Light y Singh (1987) \\
3. Identificación perceptiva de & Light y Singh (1987) \\
palabras degradadas & Mitchell (1989) \\
4. Nombrar díbujos & Moscovitch (1982) \\
5. Decisión léxica & Rabitt (1982, 1984) \\
6. Juicios de categorías & Howard (1988) \\
7. Deletrear homófonos & Light y Albertson (1989) \\
8. Asociación libre con categorías & \\
\hline
\end{tabular}

(Para más detalles, ver Light, 1991)

Parece, por tanto, que existe suficiente evidencia como para concluir que los ancianos, como los amnésicos, tienen un problema específico para tecuperar la información cuando la tarea requiere una recolección deliberada o explícita, mientras que las expresiones de priming no se ven afectadas por la edad. Dicho brevemente, el desarrollo y evolución de la memoria implícita y explícita parece ser relativamente paralelo desde la niñez hasta la vida adulta, pero en la vejez se produce un marcado declive de la memoria explícita mientras que la memoria implícita se mantiene intacta. Todo ello se manifiesta experimentalmente a través de disociaciones entre priming y memoria explícita, tanto cuando se comparan niños con adultos jóvenes y adultos jóvenes con adultos viejos, como cuando la comparación se hace entre los rendimientos del mismo grupo de ancianos en tests de priming y tests de memoria explícita. 


\subsection{Disociaciones inducidas por drogas}

Las relaciones entre drogas y memoria están siendo estudiadas exhaustivamente durante las tres últimas décadas. Aunque se ha avanzado considerablemente en el conocimiento acerca de la farmacología de la memoria (Squire y Davis, 1981), todavía resulta insuficiente el trabajo realizado para poder establecer una taxonomía de las drogas y sus efectos sobre la memoria humana (ver Idzikowski, 1988, para una revisión). No obstante, los resultados sobre los efectos de ciertas drogas (e. g., alcohol, escopolamina, anestésicos) sobre las formas implícitas y explícitas de memoria están configurando un cuadro cada vez más claro.

Por ejemplo, Hastroudi, Parker, De Lisi, Wyatt y Mutter (1984) analizaron la vulnerabilidad diferencial del priming y el recuerdo libre a los efectos del alcohol. Dos grupos de sujetos (intoxicados y sobrios) leyeron, en la fase de estudio, 29 palabras. Tras un intervalo de retención en el que realizaron varias tareas distractoras, se evaluó su retención con una prueba explícita de recuerdo libre o con una pruebra implícita de identificación de palabras degradadas. Los resultados revelaron que el efecto del alcohol sobre la memoria dependía claramente del tipo de test; a saber, los sujetos intoxicados se vieron sensiblemente afectados en el test de recuerdo libre pero, por el contrario, en el test de identificación perceptiva se beneficiaron igual que los sujetos sobrios de los efectos del priming de repetición.

Disociaciones entre priming y memoria explícita, similares a la encontrada por Hashtroudi et al. (1984) inducida por alcohol, han sido observadas por otros investigadores con el empleo de otras drogas. Nissen, Knopman y Schacter (1987) compararon el rendimiento de un grupo de sujetos a los que se había administrado una droga anticolinérgica (escopolamina) con el de un grupo de control en una amplia variedad de pruebas. Los resultados mostraron que la escopolamina había afectado sensiblemente a la ejecución de algunas tareas y no había tenido efectos significativos sobre otras. En concreto, alteró sustancialmente la ejecución de la memoria declarativa (evaluada con pruebas de recuerdo libre, recuerdo con claves y reconocimieno) y no afectó la ejecución de la memoria procedimental (evaluada con pruebas de priming de repetición). Un patrón de resultados similar ha sido encontrado por Kopelman y Corn (1988) utilizando otra droga anticolinérgica (hyoscina).

Disponemos, asimismo, de evidencia de disociaciones entre pruebas implícitas y explícitas producidas por los efectos de la anestesia general. Un número considerable de estudios ha examinado la cuestión de si se da o no memoria de los eventos que ocurren durante la anestesia general. Los resultados han sido inequívocos: la respuesta depende del tipo de prueba con la que se evalúe la retención. Por ejemplo, Roorda-Hrdlicková, Wolters, Bonke y Phaf (1990) presentaron, durante el estado de anestesia general, cuatro palabras de las categorías «frutas» (pera y banana) y «colores» (amarillo y verde) a un grupo de pacientes quirúrgicos (grupo experimental, $\mathrm{N}=43$ ) o simplemente sonidos marinos al grupo de control $(\mathrm{N}=38)$. Tres horas después de la operación, se pidió a cada uno de los pacientes que recordara lo que había ocurrido durante la anestesia. A continuación, se les pidió que dijesen los tres primeros ejemplares que les viniesen a la cabeza de las categorías «vegetales» (como ejemplo), «frutas» y «colores». Ningún paciente recordó haber oído o experimentado nada durante la anestesia. Sin embargo, en el test implícito, el grupo experimental puntuó tres veces 
más que el grupo de control (2.35 versus 0.79 ; puntuación máxima $=4)$ al generar ejemplares críticos de las categorías «frutas» y «colores». No hubo diferencias con respecto a los ejemplares generados ante la categoría no experimental «vegetales». Disociaciones similares han sido observadas por Ghoneim, Block, Ping, Ali y Hoffman (1990) y Polster, Gray, McCarthy y Park (1990).

En resumen, los datos señalan que drogas como el alcohol, la escopolamina y los anestésicos alteran la ejecución en pruebas explícitas y, sin embargo, tienen poco o ningún efecto sobre el priming. Las disociaciones entre priming y memoria explícita producidas por dichas drogas parecen, pues, similares a las producidad por la amnesia orgánica.

\subsection{Disociaciones funcionales entre priming y memoria explícita en normales}

Como ya se dijo, las disociaciones entre pruebas implícitas y explícitas de memoria no es algo exclusivo de determinadas poblaciones, sino que mediante la manipulación experimental de variables apropiadas, como puede ser el tipo de tarea de priming, las disociaciones funcionales entre priming y memoria explícita son frecuentemente producidas en sujetos normales. Jacoby y sus colaboradores (Jacoby, 1983, 1988; Jacoby y Dallas, 1981; Jacoby y Witherspoon, 1982) fueron los primeros que produjeron este fenómeno en sujetos sin ningún tipo de alteración cerebral.

En uno de sus trabajos más conocidos, Jacoby (1983) llevó a cabo una serie de experimentos con el siguiente procedimiento general. En la fase de estudio, se decía a los sujetos (estudiantes de Psicología) que se trataba de un experimento sobre los efectos del contexto en la velocidad de lectura. En esta primera fase había tres condiciones experimentales: a) Condición de No-Contexto, en la que aparecían en la pantalla una serie de $\mathrm{X}$ que, a continuación, eran reemplazadas por una palabra (e. g. XXX-FRIO). Los sujetos debían leer en voz alta y lo más rápidamente posible la palabra. b) Condición de Contexto, en la que se presentaba una palabra que era reemplazada inmediatamente por su antónimo (e. g. CALIENTE-FRIO). Los sujetos debían leer en voz alta y rápidamente la segunda palabra. c) Condición de Generar, en la que aparecía en la pantalla una palabra que era inmediatamente reemplazada por signos de interrogación (e. g. CALIENTE-???). Los sujetos debían decir en voz alta el antónimo en cuanto viesen aparecer las interrogaciones.

En las tres condiciones, como se ve, los sujetos tenían que decir la palabra crítica en voz alta, pero la respuesta se llevaba a cabo por diferentes medios. En concreto, procesando los datos en la condición de No-Contexto, procesando el significado del concepto en la condición de Generar y, presumiblemente, por una mezcla de estos dos procesos en la condición de Contexto. En todos los casos, las palabras críticas eran antónimos de las palabras del contexto.

En la fase de test, un grupo realizó una prueba de identificación perceptiva. Se presentaba una lista de palabras, de una en una, durante un breve espacio re tiempo (e. g. FRIO, durante 35 milisegundos) seguidas por una máscara (e. g. ???, durante 1 segundo). La tarea del sujeto consistía en ir diciendo en voz alta cada palabra a medida que iban apareciendo en la pantalla del taquistoscopio. Un segundo grupo recibió una prueba de reconocimiento, en la que se le proporcionaban las palabras estudiadas mezcladas con palabras nuevas y su tarea consistía en indicar aquellas que pertenecían a la fase de estudio. Los resultados pusieron de manifiesto que las palabras generadas (condición $c$ ) se habían 
reconocido mejor que las que habían sido leídas en un contexto semántico relevante (condición $b$ ) y mejor que las que habían sido leídas sin un contexto (condición a). Al mismo tiempo, se comprobó que en la prueba de identificación perceptiva el patrón de resultados de la prueba de reconocimiento aparecía invertido: el mejor rendimiento había sido el correspondiente a las palabras leídas en la condición de No-Contexto, y el peor, el resultante de la condición de General. Estos dos patrones de resultados totalmente contrapuestos encuentran su explicación en los efectos de priming producidos en la fase de estudio sobre el test de identificación perceptiva y nos ofrecen -en opinión de Roediger, Weldon y Challis (1989) - «la forma más fuerte de disociación funcional», además de poner de manifiesto que las disociaciones pueden producirse en sujetos normales.

Un elevado número de experimentos han producido disociaciones similares manipulando otras variables. Resultan de especial interés para nuestros propósitos las variables siguientes: a) el nivel de procesamiento del material de estudio; b) la relación entre el formato físico del material de la fase de estudio y el de las claves de la fase de test, y c) el intervalo temporal entre estudio y test.

a) Analicemos, en primer lugar, los datos relativos a los efectos del tipo de procesamiento sobre el priming y la memoria explícita. Por ejemplo, Jacoby y Dallas (1981) manipularon los niveles de procesamiento, a través de tareas de orientación similares a las del trabajo clásico realizado en ese marco por Craik y Tulving (1975). Durante la fase de estudio se presentaba a los sujetos una lista de palabras y, en una de las condiciones experimentales, se les hacía preguntas sobre el significado de cada palabra (tarea de orientación semántica) y, en la otra condición, se les pedía que decidiesen si la palabra contenía o no una determinada letra (tarea grafémica). En la fase de test, la retención fue evaluada con dos pruebas: una de reconocimiento y otra de identificación de palabras durante una breve presentación taquistoscópica. Tal y como se esperaba teniendo en cuenta los resultados sobre niveles de procesamiento, la codificación semántica produjo el mejor reconocimiento. Sin embargo, la maginitud de los efectos de priming fue la misma para la taea semántica y la no semántica.

Graf y Mandler (1984) encontraron un patrón similar de resultados comparando la ejecución en pruebas de recuerdo libre, de reconocimiento y de completar palabras, tras condiciones experimentales con palabras que requerían un procesamiento semántico (agradable-desagradable; significativa - no significativa; concreta-abstracta) o un procesamiento superficial (e. g. decidir si dos palabras contiguas tenían vocales en común o contar determinadas características físicas de las palabras). Los resultados fueron similares a los del estudio anterior: el recuerdo y el reconocimiento fueron significativamente superiores para la codificación semántica que para la no semántica, pero, sin embargo, los efectos de priming fueron de la misma magnitud para ambos tipos de codificación. Otros estudios, en los que se han comparado los efectos de priming sobre tareas de identificación y de completar palabras con la ejecución en pruebas explícitas, han obtenido resultados similares (e. g. Graf, Mandler y Haden, 1982; Jacoby, 1983; Roediger y Blaxton, 1987b; Schacter y Graf, 1986a; Schacter y McGlynn, 1989; Schacter, 1990a).

b) Una segunda variable de interés hace referencia a los efectos diferenciales que, sobre el priming y la memoria explícita, tienen los cambios de ciertas características superficiales de la información en la fase de test con respecto a la de estudio. Uno de los hallazgos más confirmados es que el cambio de moda- 
lidad entre estudio y test no tiene efectos significativos sobre las pruebas de memoria explícita y, por el contrario, reduce o elimina los efectos de priming. Jacoby y Dallas (1981, experimento 6) compararon los efectos del cambio de modalidad en una tarea de identificación de palabras y en una prueba de reconocimiento. Comprobaron que si se cambiaba la modalidad de la fase de estudio (auditiva) a la fase de test (visual) se producía una clara disociación: los efectos de priming se atenuaban drásticamente en la prueba de identificación perceptiva, mientras que sobre el reconocimiento no se producía efecto alguno. Graf, Shimamura y Squire (1985) obtuvieron resultados similares con pacientes amnésicos y grupos de control. En concreto, Graf et al. (1985) presentaron el material visual y auditivamente, pero las claves proporcionadas en la fase de test se presentaron siempre visualmente. La retención fue evaluada con una prueba de recuerdo libre y otra de completar raíces de palabras. Como las palabras habían sido presentadas visual y auditivamente y los fragmentos de palabras para la prueba de completar se habían presentado siempre visualmente, con esta prueba se obtenían medidas del efecto de priming tanto inter- como intramodalidad. Los resultados demostraron que el cambio de modalidad no había tenido ningún efecto sobre la prueba explícita de recuerdo libre, pero había reducido sensiblemente el rendimiento de todos los grupos en la prueba de priming de completar raíces. Schacter y Graf (1989), con un diseño factorial en el que manipularon la modalidad del input (auditiva versus visual), el tipo de contexto del test (mismo versus diferente) y el tipo de prueba (completar palabras versus recuerdo con claves), han obtenido unos resultados similares: el cambio de modalidad estudio/test redujo los efectos de priming en la tarea de completar palabras y no tuvo efectos significativos en la prueba explícita de recuerdo con claves.

Además de estos efectos de modalidad, se han observado efectos de los cambios estudio/test de otros tres tipos de características físicas. Por ejemplo, Roediger y Blaxton (1987a) estudiaron los efectos diferenciales de los cambios en la tipografía de las palabras de la fase de estudio a la fase de test. La mitad de las palabras presentadas visualmente habían sido mecanografiadas en letras minúsculas y la otra mitad habían sido escritas a mano en letras mayúsculas. La retención fue medida con una prueba implícita de completar fragmentos de palabras y una prueba explícita de reconocimiento. Tanto en una como en otra, la mitad de los fragmentos y de las palabras, respectivamente, aparecían con una tipografía mecanografiada y la otra mitad con una tipografía manual. El cambio de tipografía no tuvo efecto sobre la ejecución en la prueba de memoria explícita, pero redujo los efectos de priming en la tarea de completar palabras. Un reciente estudio de Hayman y Tulving (1989b), en el que se ha utilizado una prueba de completar palabras fragmentadas, ha demostrado, asimismo, que cambios tan pequeños como sustituir una letra por un guión y viceversa pueden tener un efecto negativo muy fuerte sobre el priming y, por el contrario, no afectar a la prueba explícita de recuerdo con claves.

Una segunda observación se refiere a los efectos diferenciales que se producen cuando la información se presenta en forma pictórica (e. g. el dibujo de un paraguas) y su retención se evalúa con pruebas que no incluyen elementos pictóricos. Por ejemplo, Weldon y Roediger (1987) presentaron 22 ítems a los sujetos, la mitad en figuras y la otra mitad en palabras. Las figuras era dibujos lineales de objetos muy comunes y las palabras eran nombres de objetos concretos y familiares también. Tras la presentación, la mitad de los sujetos realizó una prueba explícita de recuerdo libre y la otra mitad, una prueba implícita de adivinar 
palabras incompletas. Los resultados, que aparecen en la Tabla II, pusieron de manifiesto que, en la prueba de recuerdo libre, los dibujos se recordaron mejor que las palabras; sin embargo, en la prueba de adivinar palabras incompletas los efectos del priming se redujeron significativamente cuando el fragmento de la palabra que había que completar había sido presentado en forma de dibujo. Esto es, el estudio de palabras produjo un priming mayor que el estudio de dibujos.

TABLA II

Test

Estudio

Recuerdo libre

Completar fragmentos

\begin{tabular}{lcl}
\hline Dibujos & 0.35 & 0.45 \\
Palabras & 0.28 & 0.64 \\
Items nuevos & - & 0.38 \\
\hline
\end{tabular}

(Resultados de Weldon y Roediger, 1987).

La reducción e incluso la desaparición de priming cuando la presentación del material se hace con dibujos ha sido encontrada también en otros experimentos que han evaluado los efectos de priming con tests implícitos de identificación de palabras (Durso y Johnson, 1979; Kirsner, Milech y Stumpfl, 1986) y tests de decisión léxica (Scarborough, Gerard y Cortese, 1979). En contraste, y como comprobaron Weldon y Roediger (1987), la memoria explícita de palabras es mejor cuando la presentación es pictórica que cuando es verbal.

La tercera característica cuyos cambios estudio/test tienen efectos diferenciales sobre el priming y las pruebas explícitas es la lengua en la que se estudian y se evalúan los ítems. Por ejemplo, Durgunoglu y Roediger (1987) demostraron que la lengua de presentación afecta a la ejecución de los sujetos bilingües. Los sujetos (bilingües español-inglés) estudiaron 115 palabras en seis condiciones (leer cada palabra dos veces en español, leer dos veces en inglés y leer en español y en inglés) y después recibieron una de las siguientes pruebas: recuerdo libre, reconocimiento y completar fragmentos de palabras (todos los fragmentos se daban en inglés). Los resultados indicaron que los efectos de priming se habían reducido significativamente cuando el estudio se había hecho en español; es decir, cuando se había producido un cambio en la lengua de la fase de estudio a la fase de test. El recuerdo y el reconocimiento, por el contrario, no se vieron afectados. Otros estudios con sujetos bilingües han mostrado también que cuando el material se ha estudiado en una lengua y después se evalúa en otra (con pruebas de identificación, de completar fragmentos o de decisión léxica), los efectos de priming se ven reducidos sensiblemente (ver la revisión de Roediger y Blaxton, 1987a).

c) Un tercer tipo de evidencia de disociaciones funcionales entre priming y memoria explícita proviene de estudios en los que se ha manipulado el intervalo de retención entre fase de estudio y fase de test. Tulving, Schacter y Stark (1982) comprobaron que una sola presentación de una lista de 96 palabras con instrucciones de aprendizaje intencional producía un efecto de priming (medido con un test de completar fragmentos) de una magnitud que variaba ligeramente entre una prueba administrada 1 hora después del estudio a otra prueba administrada 1 semana más tarde. En concreto, las probabilidades de completar los 
fragmentos fueron de 0.47 con un intervalo de 1 hora, y de 0.46 con un intervalo de 1 semana. Esta mínima reducción contrasta fuertemente con el olvido de los mismos sujetos, revelado en su ejecución en una prueba de reconocimiento, después del mismo intervalo: la probabilidad de reconocimiento cayó de 0.78 con 1 hora de intervalo a 0.58 con 1 semana de intervalo. Otros estudios en los que se ha manipulado igualmente el intervalo de retención estudio/test han informado de similares efectos diferenciales en memoria implícita y explícita (Chandler, 1983; Komatsu y Ohta, 1984; Light et al. 1986; Roediger y Blaxton, 1987a). Recientemente, Sloman, Hayman, Ohta, Law y Tulving (1988) comprobaron la longevidad de los efectos de priming (detectados después de más de 16 meses) con pruebas de completar fragmentos de palabras frente al olvido rápido que se manifiesta en pruebas explícitas como el recuerdo y el reconocimiento.

Considerados conjuntamente, los datos presentados en este apartado sobre «disociaciones funcionales» ofrecen un cuadro convincente de que los efectos de priming en pruebas de memoria implícita difieren sustancialmente de los efectos de memoria expresados a través de las pruebas explícitas.

\subsection{Independencia estocástica entre priming y memoria explícita}

Veamos, por último, otro tipo de evidencia de disociación entre priming y recolección explícita. Se trata del hallazgo de independencia estocástica entre la ejecución en pruebas implícitas y explícitas de memoria; es decir, que el éxito o el fracaso en un test de memoria implícita no correlaciona con el éxito o el fracaso en un test de memoria explícita.

En el estudio ya referido de Tulving et al. (1982), los sujetos estudiaban una lista de 96 palabras de baja frecuencia tales como BEHAVIOR o DINOSAUR. Tras un intervalo variable, se evaluó la retención con dos tests distintos: uno explícito de reconocimiento sí/no y otro implícito de completar o rellenar fragmentos de tales palabras (e. g. el fragmento para BEHAVIOR fue BE-.VI--, y para DINOSAUR fue D-N-SA--). Aparte de los efectos diferenciales ya comentados de los dos intervalos de retención (1 hora y 1 semana) sobre ambos tipos de tests, los resultados pusieron de manifiesto que la ejecución en la prueba implícita de completar palabras no correlacionaba con la prueba explícita de reconocimiento. Es decir, cuando el test de reconocimiento precedió al test de completar palabras, los sujetos demostraron tener la misma probabilidad para completar los fragmentos de las palabras que habían reconocido que para completar los fragmentos de las palabras que no habían reconocido. Además, esta independencia estadística se observó tanto con las palabras de la lista de estudio (palabras «viejas») como con los distractores del test de reconocimiento (palabras «nuevas») y tras intervalos de 1 hora y 7 días.

Tulving et al. (1982) consideran que si las pruebas de reconocimiento y de completar palabras dependieran del mismo tipo de memoria, los sujetos deberían haber completado más fragmentos de palabras reconocidas previamente que de palabras no reconocidas; es decir, que la ejecución debería haber correlacionado positivamente en ambas pruebas, pero, sin embargo, no fue así. Por tanto, «nuestros datos sugieren que... la información que los sujetos utilizan para completar los fragmentos de las palabras facilitadas (primed) no es el mismo tipo de información con el que la gente cuenta para rememorar eventos de su pasado» (Tulving et al., 1982, p. 341; ver también Tulving, 1983).

Este tipo de independencia ha sido encontrado también entre tests de reco- 
nocimiento y tests de deletreo de homófonos (Jacoby y Witherspoon, 1982; Eich, 1984), de completar raíces (Graf y Schacter, 1985) y de decisión de objetos (Schacter, Cooper y Delaney, 1990a). En todos estos estudios se ha comprobado que la buena ejecución en pruebas de memoria implícita no correlacionaba con el éxito o el fracaso en una prueba de memoria explícita. No obstante, algunos críticos han argumentado que la independencia estocástica podría ser un artificio producido por la influencia del primer test sobre el segundo (e. g. Shimamura, 1985; Mandler, Graf y Kraft, 1986; ver la revisión de Richardson-Klavehn y Bjork, 1988). La lógica de la independencia estocástica supone que un sujeto concreto sea evaluado en el mismo ítem con una prueba explícita y una prueba implícita. Entonces, como señalan Richardson-Klavehn y Bjork (1988), resulta plausible suponer que las presentaciones de un ítem y las reacciones del sujeto que supone la realización de la primera prueba introduzcan alguna alteración en el sistema de memoria que contaminará la ejecución en la segunda. Sin embargo, el reciente trabajo de Schacter et al. (1990a) parece eludir tales efectos ya que sus resultados indican que la independencia estocástica fue observada incluso cuando no hubo evidencia de efectos de priming producidos por el test. No obstante, Schacter et al. (1990a) reconoce que no siempre es sencillo interpretar un hallazgo de independencia estocástica por el hecho de que, a veces, puede aparecer entrubiado por una variedad de cuestiones sutiles y complejas (ver la revisión de Shimamura, 1985, y, sobre todo, las discusiones de Hayman y Tulving, 1989a, b).

\section{3. ¿QUE SIGNIFICAN LAS DISOCIACIONES? PROPUESTAS TEORICAS}

En los últimos años se ha hecho patente el recuerdo de que resulta adecuado distinguir distintas formas de memoria. Tulving $(1972,1983)$ distinguió entre memoria episódica y semántica; Graf y Schacter (1985) dintinguen entre memoria implícita y explícita; Tulving (1985) modifica su primera clasificación y considera más adecuado diferenciar entre memoria procedimental, memoria semántica y memoria episódica; Squire $(1986,1987)$ distingue entre memoria declarativa y procedimental, y, muy recientemente, Tulving y Schacter (1990) proponen distinguir entre priming, memoria procedimental, memoria semántica y memoria episódica. Ahora bien, este acuerdo en cuanto a clasificar la memoria en formas distintas se rompe a la hora de establecer las bases teóricas y empíricas que se supone dan sustento a tales clasificaciones. En este sentido, las abundantes disociaciones encontradas entre pruebas implícitas y explícitas de memoria han sido consideradas por un elevado número de investigadores como una prueba firme de la validez de tales tipologías, llegando a postular la existencia de diferentes sistemas de memoria (e. g. Cohen y Squire, 1980; Tulving, 1983, 1985, 1987; Johnson, 1983; Schacter, 1987, 1989, 1990a; Squire, 1986, 1987; Sherry y Schacter, 1987; Weiskrantz, 1987, 1989; Tulving y Schacter, 1990). Para otros, sin embargo, las disociaciones entre memoria implícita y explícita son conceptualizadas más adecuadamente en términos de diferentes procesos que operan dentro de un único sistema de memoria (e. g. Jacoby, 1983, 1988; Graf y Mandler, 1984; Moscovitch, Winocur y McLachlan, 1986; Roediger y Blaxton, 1987a; Roediger y Weldon, 1987; Roediger, Weldon y Challis, 1989; Roediger, Rajaram y Srinivas, 1990). 
La evidencia presentada en el apartado anterior sobre disociaciones entre priming y memoria explícita es tan impresionante que su interpretación teórica se ha convertido en los últimos años en uno de los temas que más interés y atención está recibiendo, al tiempo que ha generado un debate formidable sobre los mecanismos subyacentes. Se han propuesto distintas explicaciones pero, como veremos, todas ellas resultan por sí solas insuficientes para poder explicar los abundantes datos disponibles. Ahora bien, los avances que se están produciendo en el plano experimental están siendo tan rápidos que la necesidad de revisar las ideas teóricas se ha hecho igualmente imperiosa. Ante la rápida sucesión de propuestas, matizaciones, modificaciones y contrapropuestas, no resulta fácil ofrecer un cuadro claro del estado actual de las cosas. Por todo ello, y con el propósito de hacer lo más comprensible posible la breve pero intensa historia de las disociaciones entre priming y memoria explícita y de sus explicaciones, nuestra exposición seguirá una cierta cronología. Así pues, en primer lugar, expondremos los enfoques que, hasta hace relativamente poquísimos años, han configurado la doctrina oficial al respecto. A continuación comentaremos algunos de los intentos por integrar las dos propuestas explicativas más consistentes. En tercer y último lugar, presentaremos datos muy recientes, procedentes de estudios que incorporan ideas de neuropsicología cognitiva, que proponen que el priming es la expresión de un sistema de representación perceptual y, como tal, puede considerarse como una forma no - consciente de memoria distinta de otras formas de memoria conocidas.

\subsection{Propuestas explicativas de las disociaciones entre priming y memoria explícita}

En la década de 1980 surgieron distintas explicaciones para las disociaciones que son objeto de nuestro trabajo (ver revisiones de Schacter, 1987, y Richardson-Klavehn y Bjork, 1988). Schacter (1987) se refirió a ellas en estos términos: activación, procesamiento y sistemas múltiples de memoria. Durante toda la década pasada, las explicaciones en términos de estas tres propuestas han configurado el panorama teórico dominante y entre ellas se ha desarrollado y mantenido un acalorado debate.

Por otra parte, Richardson-Klavehn y Bjork (1988) consideran que las relaciones entre memoria implícita y explícita han sido abordadas desde unos presupuestos teóricos que permiten identificar, entre otras, tres posiciones: la abstraccionista, la no - abstraccionista y la híbrida.

El enfoque abstraccionista considera que la memoria implícita refleja la modificación de estructuras abstractas de conocimiento léxico, semántico o procedimental, mientras que la memoria explícita se considera que depende de la formación y recuperación de huellas de memoria de experiencias concretas. Es esta distinción entre representaciones abstractas y huellas de memoria que representan eventos específicos lo que caracteriza a esta posición. Los abstraccionistas son mayoritariamente teóricos de orientación neurocientífica y básicamente manejan datos de pacientes con lesiones cerebrales (e. g. Warrington y Weiskrantz, 1982; Cohen y Squire, 1980; Cohen, 1984; Squire y Cohen, 1984; Squire, 1986, 1987). Las disociaciones entre tests de memoria implícita y explícita son explicadas en términos de diferentes sistemas de memoria: declarativa versus procedimental (Cohen y Squire, 1980; Squire, 1986, 1987) o sistema de memoria versus sistema de bábitos (Mishkin, Malamut y Bachevalier, 1984). No 
obstante, aquí también estarían incluidas las propuestas de psicólogos cognitivos como Tulving $(1972,1983,1985)$ con su teoría monojerárquica de la memoria, que distingue entre el sistema de memoria procedimental, el sistema de memoria semántica y el sistema de memoria episódica, y que explicaría las disociaciones en base a estos tres sistemas; Morton (1969, 1979; Morton y Patterson, 1980) con su modelo de logogenes, que explicaría las disociaciones en base a las representaciones de un sistema cognitivo y diferentes sistemas de logogenes (visual y auditivo), o Mandler (1980, 1989; Graf y Mandler, 1984) — neutral con respecto a la cuestión de diferentes sistemas de memoria- que explicaría las disociaciones apelando a los procesos de activación y elaboración.

El enfoque no abstraccionista está representado por psicólogos cognitivos exclusivamente. Estos no están de acuerdo con la distinción entre representaciones abstractas y huellas de memoria de eventos específicos. Tampoco aceptan la distinción entre conocimiento declarativo y procedimental, argumentando que las proposiciones o declaraciones también contienen representaciones procedimentales (Kolers y Roediger, 1984). Las disociaciones entre pruebas implícitas y explícitas son explicadas en términos de solapamiento entre las operaciones de codificación y las de recuperación. Por tanto, no consideran necesario postular diferentes sistemas de memoria para explicar las disociaciones. Las demandas de procesamiento de los tests de memoria (guiados conceptualmente vs. guiados por los datos) estarían en la base de tales disociaciones (Jacoby, 1983; Roediger y Blaxton, 1987a, b; Roediger, Weldon y Challis, 1989; Roediger, 1990a).

La posición bibrida acepta, como los abstraccionistas, la distinción entre representaciones abstractas y huellas de memoria que representan experiencias; sin embargo, asume un acceso dual a la memoria episódica. A esta memoria puede accederse tanto explícitamente (con conciencia) como implícitamente (sin conciencia) (Feustel, Shiffrin y Salasoo, 1983; Salasoo, Shiffrin y Feustel, 1985; Squire, Shimamura y Graf, 1987). Las disociaciones entre pruebas implícitas y explícitas son explicadas en términos de un acceso no consciente o consciente, respectivamente, a la información episódica. Las diferentes claves proporcionadas por los tests son las que determinan el tipo de acceso a la memoria episódica.

Pero, como señalábamos un poco más arriba, el debate teórico sobre el significado de las disociaciones se ha planteado en los términos señalados por Schacter (1987). Por tanto, consideramos pertinente exponerlo aquí en base a las explicaciones que hacen referencia a la activación, sistemas múltiples de memoria y procesamiento. No se trata de repetir lo que acabamos de decir al respecto, sino de detenernos en aquellos aspectos que por su relevancia teórica mejor explican el fenómeno de las disociaciones.

\subsubsection{Explicaciones en términos de activación}

El argumento central de esta propuesta es que determinadas representaciones de memoria (como, por ejemplo, las palabras) se activan automáticamente con la mera presentación en la fase de estudio (Mandler, 1980, 1989; Morton, 1969; Graf y Mandler, 1984). Hayes-Roth (1977) describió tales representaciones mnésicas como estructuras integradas en unidades («estructuras unitizadas»), de tal manera que cualquier unidad o toda ella puede actuar para reintegrar todo el estímulo. En base a ello, la teoría de la activación sugiere que sólo los 
ítems con representaciones previamente almacenadas producirán efectos de priming dado que tales ítems son los únicos que cuentan con representaciones unitizadas. Sin embargo, este presupuesto teórico cuenta con fuerte evidencia en su contra desde el momento en que se han demostrado efectos de priming tanto para palabras como para no - palabras (que no tienen representaciones mnésicas pre - almacenadas) (Feustel et al., 1983; Salasoo et al., 1985). Además, en diferentes estudios se encontraron efectos de priming de pares asociados nuevos (e. g. VENTANA-RAZON) (Graf y Schacter, 1985, 1989; Schacter, 1985; Schacter y Graf, 1986a, 1989).

El otro presupuesto básico de esta teoría es que el priming no requiere un procesamiento elaborativo. Graf y Mandler (1984) describieron la elaboración como un proceso «requerido para percibir relaciones entre un conjunto de palabras previamente no relacionadas» (p. 554). Por tanto, para que se manifieste el priming sólo se necesita activación; mientras que la recuperación explícita necesitará un procesamiento de elaboración. Pues bien, también este presupuesto ha sido desafiado por estudios en los que se comprobó que el priming se producirá sólo cuando los sujetos procesaban elaborativamente los pares de palabras (Graf y Schacter, 1985; Schacter y Graf, 1986a), por ejemplo, generando una oración que relacionaba las dos palabras.

Este enfoque es consistente, no obstante, con los siguientes hallazgos: a) el priming de representaciones preexistentes no depende del procesamiento elaborativo (e. g. Jacoby y Dallas, 1981; Graf, Mandler y Haden, 1982); b) algunos pacientes amnésicos que muestran efecto de priming de ítems familiares no muestran priming de no - palabras ni de pares asociados no relacionados (e. g. Diamond y Rozin, 1984; Cermak et al., 1985; Schacter y Graf, 1986b), y c) bajo ciertas condiciones, el priming decae rápidamente tanto en normales como en amnésicos (Graf y Mandler, 1984; Shimamura y Squire, 1984; Cermak et al., 1985).

El presupuesto general de que el priming está mediatizado por la activación de representaciones mnésicas preexistentes es asumido, también, por el modelo de logogenes de Morton (1969, 1979). Sin embargo, estamos de acuerdo con Schacter (1990a) al considerar a esta propuesta como un ejemplar de explicación en términos de sistemas múltiples de memoria. En consecuencia, será tratada dentro del apartado siguiente.

\subsubsection{Explicaciones en términos de sistemas múltiples de memoria}

El fenómeno de priming de repetición encontró una de sus primeras explicaciones en el modelo de logogenes de Morton (1969, 1979; Morton y Patterson, 1980). En su primera formulación, Morton (1969) consideró a los logogenes como unidades léxicas abstractas independientes de la modalidad, que se activan con la presentación de una palabra. La activación de un logogén rebaja temporalmente su umbral, por lo que la presentación de una palabra facilitará su detección en un test posterior de identificación. Sin embargo, este primer modelo no podía explicar los efectos de priming específicos de la modalidad, así que tuvo que ser reformulado en términos de sistemas separados de logogenes (Morton, 1979). Con la distinción de un sistema visual (que contiene representaciones de la forma visual de las palabras) y un sistema auditivo (que contiene representaciones de la forma hablada de las palabras), Morton (1979) pudo acomodar el fenómeno de priming específico de la modalidad. Además de estos dos 
sistemas, Morton distinguió un «sistema cognitivo» que es independiente de los sistemas de logogenes y que es responsable del procesamiento semántico y del recuerdo episódico o explícito. La nueva formulación de Morton (1979) puede considerarse, pues, como un ejemplo de sistemas separados de memoria, en el que se distinguen tres sistemas distintos: dos sistemas de logogenes (visual y auditivo), implicados en los efectos de priming o memoria implícita, y un sistema cognitivo involucrado en la recolección explícita.

Sin embargo, este nuevo modelo de Morton (1979) se ha encontrado con otros problemas de difícil solución. A saber, los efectos negativos que sobre el priming tienen los cambios en las características superficiales de la información entre la fase de estudio y la fase de test. Recuérdese, por ejemplo, que distintos estudios han mostrado que pequeños cambios en la tipografía reducen o incluso anulan el priming (e. g. Roediger y Blaxton, 1987a; Hayman y Tulving, 1989b). Si el logogén visual se considera como una representación abstracta y preexistente de la forma visual de la palabra, los efectos de priming no deberían verse afectados por tales cambios. Además, este enfoque también resulta insuficiente para explicar el hecho comprobado de que los efectos de priming pueden tener una larga duración (e. g. Tulving et al., 1982; Sloman et al., 1988) ya que postula que la activación de los logogenes decae en segundos o minutos (e. g. Roediger y Blaxton, 1987a).

Parece razonable, pues, aceptar que este modelo resulta insuficiente para explicar las disociaciones entre pruebas de memoria implícita y explícita por su incapacidad para dar cobertura teórica a la biperespecificidad y la persistencia temporal de ciertos fenómenos de priming (Schacter, 1990a).

Pero la propuesta que mejor representa la explicación de las disociaciones en términos de distintos sistemas de memoria es la de aquellos autores que han apelado a las propiedades diferentes de hipotéticos sistemas mnésicos. Entendiendo por sistemas de memoria «estructuras organizadas de componentes operantes más elementales» (Tulving, 1985) o «una interacción entre mecanismos de adquisición, retención y recuperación que se caracteriza por ciertas reglas de funcionamiento» (Sherry y Schacter, 1987, p. 440). Ahora bien, mientras que en neurociencia la modularidad de los sistemas es la regla más que la excepción, en psicología cognitiva la idea de sistemas de memoria como módulos independientes (Fodor, 1983) tiene al menos dos versiones: 1) una versión fuerte, que considera al sistema de memoria como «una unidad funcionalmente autónoma en la que los procesos componentes interactúan exclusivamente entre sí y operan con independencia de las otras unidades» (Sherry y Schacter, 1987, p. 440), y 2) una versión débil, según la cual «un sistema de memoria es una interacción entre los procesos de adquisición, retención y recuperación en la que cualquiera de tales componentes puede interactuar con otros procesos externos al sistema» (op. cit., p. 440). Si bien los investigadores del ámbito de la neurociencia no tienen inconveniente alguno en aceptar y utilizar la versión fuerte, la mayoría de los psicólogos cognitivos de la memoria se decantarían más - al menos por el momento- por la versión débil.

Como ya se comentó al hablar de la posición abstraccionista, varios investigadores del campo de la neurociencia han argumentado que la recolección consciente o explícita es una propiedad de un sistema de memoria declarativa, mientras que los fenómenos de memoria implícita tales como el priming directo y el aprendizaje de habilidades reflejan las operaciones de un sistema de memoria procedimental (Cohen, 1984; Squire, 1986, 1987). La memoria procedimental se 
considera que está ligada a y se expresa a través de la activación de estructuras particulares de procesamiento implicadas en las tareas de aprendizaje, y se adquiere y se retiene en virtud de la plasticidad inherente de esas estructuras. Sus efectos se consideran reflejo de modificaciones on-line de los procesos de codificación (Squire, 1987). Por el contrario, la memoria declarativa es considerada como más cognitiva, rápida y adaptada para el aprendizaje de ensayo único y está implicada en el almacenamiento de huellas que representan eventos particulares que sucedieron en un momento y un lugar concreto. Sus efectos, es decir, la recolección explícita, dependen de las representaciones resultantes de los procesos de codificación (Squire, 1987).

Otros investigadores han apelado a la distinción entre memoria episódica y semántica para explicar las disociaciones entre priming y memoria explícita (e. g. Schacter y Tulving, 1982; Tulving, 1983; Cermak et al., 1985). La memoria semántica serviría de base a la ejecución en tareas implícitas tales como completar e identificar palabras, en las que el sujeto tiene que recurrir al conocimiento preexistente sobre tales palabras. La memoria episódica, por su parte, es considerada la responsable del recuerdo y el reconocimiento explícitos. Algunos de los proponentes de estas explicaciones han sugerido también que los efectos de priming reflejan las operaciones de un «sistema cuasi-mnésico sin huellas» que no operaría sobre huellas o representaciones de memoria (Tulving, 1983, 1985; Hayman y Tulving, 1989a, b; volveremos sobre esta hipótesis más adelante). Otras muchas explicaciones de las disociaciones entre priming y memoria explícita han sido planteadas también en términos de sistemas múltiples de memoria (e. g. Johnson, 1983; Schacter y Moscovitch, 1984; Tulving, 1985; Sherry y Schacter, 1987; Mitchell y Brown, 1988; Weiskrantz, 1987, 1989; Schacter, McAndrews y Moscovitch, 1988; Tulving y Schacter, 1990; Schacter, 1989, 1990a, c).

Uno de los argumentos más fuertes con los que cuenta este enfoque en su favor es que, con la distinción entre sistemas múltiples de memoria (declarativa y procedimental; episódica, semántica y procedimental, etc.), ofrece una buena explicación de la ejecución típica de los pacientes amnésicos: memoria implícita preservada a pesar de su incapacidad para rememorar (reconocer o recordar) conscientemente o explícitamente las experiencias previas de aprendizaje. Sin embargo, esta hipótesis resulta insuficiente para explicar el hecho de que algunos amnésicos no muestran efectos de priming para las no - palabras y la información nueva (e. g. Diamond y Rozin, 1984; Cermak et al., 1985; Graf y Schacter, 1985; Schacter y Graf, 1986b; Moscovitch et al., 1986). Como señala Schacter (1987), si el priming refleja la modificación de los procedimientos utilizados para codificar los estímulos, se debería producir tanto con la información vieja como con la nueva. Por otra parte, los datos sobre efectos de priming en sujetos normales no tienen por qué ser considerados como una prueba decisiva de la existencia de múltiples sistemas de memoria. De hecho, el tipo de independencia funcional encontrado entre priming y pruebas de memoria explícita también ha sido encontrado en el dominio de la memoria explícita, donde no hay necesidad de apelar a la operación de sistemas diferentes de memoria (Roediger, 1984). Además, Witherspoon y Moscovitch (1989) han encontrado independencia estocástica entre dos tests implícitos (completar fragmentos e identificación perceptual), aunque tal hallazgo haya sido matizado con una elaboradísima metodología experimental por Hayman y Tulving (1989a, b). No parece necesario, por tanto, en opinión de los teóricos del enfoque de procesamiento, postular la existencia de sistemas separados de memoria para explicar los datos. 


\section{4}

\subsubsection{Explicaciones en términos de procesamiento}

En contraste con la explicación en términos de sistemas múltiples, este tercer enfoque explica las disociaciones entre pruebas implícitas y explícitas apelando a la naturaleza y a la relaciones entre los procesos de codificación y recuperación. Dicho de otra manera, las disociaciones podrían reflejar la operación de los distintos procedimientos cognitivos requeridos por los tests de memoria (Roediger y Blaxton, 1987a; Roediger y Weldon, 1987; Roediger, Weldon y Challis, 1989; Roediger, 1990a). Los proponentes de este enfoque mantienen que tanto la expresión implícita como la explícita de la memoria están basadas en representaciones episódicas de nueva creación dentro de un sistema unitario de memoria. Por tanto, en su opinión, ni es necesario postular sistemas separados para explicar las disociaciones, ni la estrategia de identificar una tarea con un sistema concreto ayuda a esclarecer la naturaleza del fenómeno.

La suposición central de este enfoque de procesamiento es que las pruebas de memoria se basan en procesos diferentes y que lo que reflejan las disociaciones entre ellas es las operaciones de tales procesos. Roediger (1990a) ha resumido recientemente las ideas básicas de este enfoque en los siguientes cuatro puntos: 1) la ejecución en pruebas de memoria depende del grado de solapamiento entre las operaciones cognitivas de la fase de test y las de la fase de estudio. Este argumento, que sería aceptado por cualquier teórico con independencia de sus ideas, se asemeja a «la hipótesis de codificación específica» (Tulving y Thomson, 1973), aunque los supuestos de procesamiento son ligeramente diferentes (Roediger et al., 1989). En concreto, se basa en las ideas de «transferencia apropiada de procesamiento» (Morris, Bransford y Franks, 1977), en el punto de vista procedimental (Kolers y Roediger, 1984) y en la distinción entre tests introducida por Jacoby (1983). 2) Las pruebas, según sean explícitas o implícitas, generalmente (aunque no siempre) necesitan operaciones diferentes de recuperación o de acceso a la información; consecuentemente, se benefician diferencialmente de los tipos de procesamiento que se llevan a cabo durante la fase de estudio. Este supuesto se entiende cabalmente teniendo en cuenta los dos siguientes. 3) La ejecución en la mayor parte de las pruebas explícitas depende de la codificación o el procesamiento elaborativo, del significado codificado de conceptos, etc. De ahí que las pruebas explícitas sean muy sensibles a la elaboración conceptual e insensibles a los cambios que se producen en las características superficiales de la información. 4) La ejecución en la mayor parte de las pruebas implícitas depende de la comparación entre operaciones perceptuales entre las fases de estudio y test. Por esta razón, estas pruebas son muy sensibles a los cambios de la forma superficial de la información (e. g. cambios de modalidad, tipográficos, etc., entre estudio y test) y relativamente insensibles al nivel de elaboración del procesamiento (e. g. Jacoby y Dallas, 1981; Graf y Mandler, 1984).

En base a ello, Jacoby (1983) ha introducido la distinción entre tests guiados conceptualmente o que reflejan el grado de elaboración conceptual, y tests guiados por los datos o que reflejan un procesamiento perceptual. Aunque tanto los tests implícitos como los explícitos pueden tener componentes guiados conceptualmente y guiados por los datos, se ha argumentado que los tests de memoria explícita se basan generalmente en procesos guiados conceptualmente, mientras que los tests de memoria implícita se basan generalmente en procesos guiados por los datos (Jacoby, 1983; Roediger y Blaxton, 1987a). A partir de estas ideas, 


\section{5}

las disociaciones entre pruebas implícitas y explícitas son atribuidas a diferencias entre procesos guiados conceptualmente y guiados por los datos (Roediger y Blaxton, 1987a; Blaxton, 1989).

Esta propuesta, al mantener que tanto la memoria explícita como la implícita tienen una base episódica, cuenta en su favor con las observaciones de persistencia, efectos asociativos, sensibilidad contextual e interacciones estudio/test (Jacoby, 1983; Roediger y Blaxton, 1987a). Sin embargo, tiene serias dificultades para explicar hallazgos tales como la activación de corta duración, la dependencia de algunos efectos de priming de representaciones preexistentes en pacientes amnésicos y las diferencias entre priming de nuevas y viejas representaciones en sujetos normales (e. g. Schacter y Graf, 1986a). Además, este enfoque no dice nada acerca de la característica clave de los fenómenos de memoria implícita: la ausencia de recolección consciente de un episodio previo.

Pero ante todo, el problema fundamental con el que se enfrenta el enfoque de procesamiento es que no ofrece una explicación satisfactoria del hecho repetidamente comprobado de que la memoria implícita se mantiene preservada en los pacientes amnésicos. Este problema, como ha señalado muy recientemente Schacter (1990a) y ha reconocido explícitamente Roediger (1990a), es particularmente importante porque el hecho que encierra constituye probablemente la base más importante para la distinción entre memoria implícita y explícita. Una posibilidad - desde el punto de vista de los teóricos de procesamiento- sería que los amnésicos son deficientes para llevar a cabo un procesamiento guiado conceptualmente. Pero no hay ninguna prueba de que esto ocurra (ver Roediger, 1990a). Es más, hay datos que muestran que los pacientes amnésicos muestran efectos intactos de priming en pruebas implícitas que parecen depender claramente de procesos guiados conceptualmente, como por ejemplo, la producción de ejemplares de categorías (e. g. Graf, Shimamura y Squire, 1985; Shimamura, 1986) y la asociación libre (e. g. Shimamura y Squire, 1984; Schacter, 1985; McAndrews, Glisky y Schacter, 1987).

En resumen, la valoración global de las tres explicaciones expuestas señala que todas y cada una de ellas cuenta con ciertos datos a favor pero, al mismo tiempo, con dificultades para explicar otros datos. Es decir, que por sí solas ninguna de tales explicaciones resulta teóricamente suficiente para dar cuenta satisfactoria de todos los hallazgos relativos a los efectos de priming. $\mathrm{Y}$ este es, en esencia, el problema que hay que explicar.

No obstante, la falta de acuerdo no ha sido obstáculo, sino todo lo contrario, para que en los últimos pocos años se haya avanzado con una especial rapidez en la comprensión de la naturaleza del priming. En nuestra opinión, ello ha sido posible gracias, fundamentalmente, a la labor intensa y rigurosa del grupo de trabajo de Endel Tulving (Hayman y Tulving, 1989a, b; Tulving y Schacter, 1990) y de Daniel Schacter (1989, 1990a, c; Schacter et al., 1989; Schacter, Cooper y Delaney, 1990a y b; Schacter, Delaney y Merikle, 1990; Schacter et al., 1991) que, introduciendo nuevas y sofisticadas metodologías (ver Hayman y Tulving, 1989a) e incorporando ideas de otras disciplinas como la neuropsicología cognitiva (Schacter et al., 1988; Schacter, Cooper y Delaney, 1990a y b; Schacter et al., 1991; Schacter, 1990a, c; Tulving y Schacter, 1990), han conseguido dos objetivos que hasta hace muy poco parecían inalcanzables: 1) ofrecer una solución al conflicto entre el enfoque de procesamiento y el de sistemas múltiples de memoria mediante una propuesta teórica integradora, y 2) presentar una alternativa teórica que explica y define coherentemente la naturaleza y la arqui- 
tectura del sistema representacional en el que se fundamentan los efectos de priming.

\section{INTENTOS DE INTEGRACION}

En el trabajo más reciente de Roediger (1990a), el proponente por excelencia del enfoque de procesamiento, se reconocen no sólo las insuficiencias de este enfoque para explicar adecuadamente algunos hallazgos (en especial el hecho comprobado de que los amnésicos exhiben un priming preservado en muchos tests implícitos que parecen estar guiados conceptualmente), sino también que la propuesta de sistemas múltiples de memoria no deja de recibir apoyo de la neuropsicología cognitiva. No obstante, sigue admitiendo que los datos de sujetos normales encuentran la mejor explicación en las ideas de transferencia apropiada de procedimientos, mientras que los datos neuropsicológicos y psicobiológicos se explican más adecuadamente apelando a diferentes sistemas de memoria. Consecuentemente, en su opinión, la explicación de los efectos de priming continúa sin salir del impasse en el que se encuentra desde comienzos de la pasada década.

Esta, por el contrario, no es la opinión actual de Tulving y Schacter (1990; Schacter, 1990a), para quienes no tiene por qué haber una incompatibilidad necesaria entre la propuesta de procesamiento y la de sistemas de memoria, sino que es posible $-e$ incluso necesaria- una integración entre ambos enfoques. Después de todo, es necesario apelar a procesos para que los sistemas funcionen. El primer paso para tal integración ha sido dado por Hayman y Tulving (1989a) al incorporar las ideas básicas del enfoque de procesamiento a la propuesta de sistemas múltiples.

En su constante búsqueda de una explicación a los efectos de priming, Tulving (Hayman y Tulving, 1989a, b) sugiere que tales efectos dependen de un sistema cerebral-cognitivo, distinto del que da soporte a la memoria episódica, al que llama «sistema $\mathrm{QM}$ » (cuasi-mnésico). En realidad, la idea de que los efectos de priming deber ser considerados como un fenómeno independiente de la memoria episódica y no asimilable a la memoria semántica ya se encuentra en un trabajo muy anterior (Tulving, Schacter y Stark, 1982; Tulving, 1983). En aquella primera ocasión, Tulving et al. (1982, p. 341) expresaron esta hipótesis con claridad, aunque con reservas, así: «Dado que lo efectos de priming... son independientes de la memoria episódica, y puesto que hay problemas para interpretarlos en términos de modificaciones de la memoria semántica, nos tienta pensar que éstos reflejan la operación de algún otro, todavía poco conocido, sistema de memoria». La hipótesis del «sistema $\mathrm{QM}$ » - que será reelaborada en un trabajo más reciente (Tulving y Schacter, 1990) — permite incorporar muchas de las ideas contenidas en el enfoque de procesamiento. Según Hayman y Tulving (1989a), algunas de las propiedades funcionales del «sistema $\mathrm{QM} \gg$ han sido descritas por Roediger et al. (1989) como argumentos en apoyo del enfoque de procesamiento. Tales propiedades incluirían: a) sensibilidad a las características superficiales de la información procesada y falta relativa de sensibilidad a las características semánticas, y b) resistencia al olvido y a la interferencia. Parece, pues, teóricicamente coherente suponer que los efectos del «sistema QM» están basados en información y procesos de recuperación diferentes de los que sustentan a los fenómenos de memoria episódica. Todo ello hace al «sistema QM» especialmente adecuado para explicar el hecho de que los pacientes amnésicos presentan una 
memoria episódica alterada al tiempo que muestran unos efectos de priming normales o casi normales. De hecho, un sistema con tales propiedades resulta capaz de explicar la mayor parte de los hallazgos empíricos presentados a lo largo de este trabajo, como, por ejemplo: 1) la sensibilidad de los efectos de priming a las características superficiales de los estímulos; 2) la longevidad de los efectos de priming; 3) el desarrollo temprano de los efectos de priming en los niños; 4) la resistencia de los efectos de priming al envejecimiento natural; 5) las disociaciones encontradas en los experimentos con pacientes amnésicos, y 6) las disociaciones inducidas por drogas. Sin embargo, el «sistema $Q M »$ presenta una gran debilidad, y es que la explicación que ofrece del priming es una explicación «pos hoc». En opinión de sus proponentes, puede acomodar los datos pero no permite hacer predicciones.

Para resolver esta insuficiencia, Hayman y Tulving (1989b) analizan con toda precisión las condiciones bajo las que se produce y no se produce independencia estocástica entre dos pruebas implícitas (Tulving et al., 1982; Witherspoon y Moscovitch, 1989; y especialmente Hayman y Tulving, 1989b) y concluyen que dichos hallazgos reflejan la ausencia de huellas de memoria sobre las que operarían los procesos de recuperación del «sistema $Q M »$, por lo que entienden que éste podría ser considerado como un sistema «sin huellas». El aprendizaje en el «sistema QM sin huellas» podría entenderse en términos de «fortalecimiento con conexiones concretas estímulo-respuesta más que en términos de recuperación o de acceso a información que representa un estado del mundo no presente perceptualmente», como es el caso de la información almacenada en la memoria episódica y semántica (Hayman y Tulving, 1989b, p. 953). Por otra parte, los efectos de priming en este sistema reflejarían la utilización de procedimientos específicos reforzados, pero «no reflejarían ningún almacenamiento ni modificación en el almacenamiento de información proposicional relativa a estímulos o eventos» (op. cit., p. 953). En consecuencia, el «sistema QM sin huellas» es considerado semejante o análogo a la memoria procedimental o a un subsistema de ésta, y los efectos de priming comparables a los cambios en los procedimientos internos a los que se refieren Kolers y Roediger (1984); es decir, podrían considerarse como un ajuste de procedimientos específicos dentro de un sistema de memoria QM sin huellas.

Por todo ello, Hayman y Tulving (1989a, b) proponen atribuir los efectos de priming a un sistema cuasi-mnésico (QM) que no contiene huellas integradas de estímulos, eventos o hechos del tipo de los representados en los sistemas de memoria episódica y semántica, y que estaría relacionado con el sistema de memoria procedimental o sería un subsistema de ésta. Es por ello que los efectos de priming no son accesibles a la introspección ni permiten establecer juicios sobre los contenidos «ecforizados» de la memoria episódica. En su opinión, un sistema así es perfectamente integrable con las ideas de los teóricos de procesamiento que explican los efectos de priming en base a una transferencia apropiada de procedimientos.

Sin embargo, la idea de asimilar el sistema QM a la memoria procedimental ha sido seriamente desafiada por evidencia reciente de disociaciones entre pruebas de memoria implícita en diferentes poblaciones de pacientes neurológicos. En dos estudios (Heindel et al., 1989; Butters et al., 1990) en los que se comparó la ejecución de pacientes con enfermedad de Alzheimer y pacientes con enfermedad de Huntington en diferentes tareas de memoria implícita y explícita, se observaron patrones de resultados prácticamente idénticos: una clara alteración 


\section{8}

en las tareas de memoria explícita y marcadas disociaciones en tareas implícitas. Pero en lo que concierne a estas últimas, se comprobó que 1) en tareas de priming (léxico y pictórico), los pacientes de Alzheimer mostraban una alteración severa, mientras que los pacientes de Huntington demostraban una capacidad normal, y 2) en tareas de aprendizaje de habilidades motoras se observó la relación opuesta. Estas disociaciones dobles entre pacientes de Alzheimer y de Huntington en tareas de priming y aprendizaje de habilidades han sido interpretadas como prueba de que dos formas de memoria implícita - que están intactas en pacientes amnésicos - no dependen de la misma constelación de estructuras cerebrales. Los datos parecen indicar, por el contrario, que hay al menos dos sistemas de memoria implícita (el sistema responsable de los efectos de priming y el sistema de memoria procedimental) que son distintos tanto neurológica como psicológicamente (Butters et al., 1990). La no dependencia del priming con respecto a la memoria procedimental es asumida por Tulving en su último trabajo (Tulving y Schacter, 1990), que será comentado más adelante.

Llegados a este punto, resulta obligado hacerse la siguiente pregunta: si los efectos de priming parecen ser independientes del sistema de memoria episódica, del sistema de memoria semántica y del sistema de memoria procedimental, ¿qué tipo de memoria es el priming? o ¿qué sistema representacional, de aprendizaje o de memoria subyace a los efectos de priming?

\section{LA NATURALEZA DEL PRIMING}

En un intento por resolver el debate planteado desde medidados de la pasada década entre el enfoque de procesamiento y el de los sistemas múltiples de memoria, Schacter (1990a) ha sugerido que los efectos de priming observados en una variedad de pruebas de memoria implícita (específicamente, las que dependen de un priming perceptual) son el reflejo de la actividad de un tipo de procesadores o subsistemas modulares que juntos forman el sistema de representación perceptual o PRS (Schacter, Cooper y Delaney, 1990a, b; Schacter, Delany y Merikle, 1990; Schacter, Cooper, Delany, Peterson y Tharan, 1991; Tulving y Schacter, 1990). Estos procesadores o subsistemas modulares han sido identificados en estudios neuropsicológicos recientes sobre déficits perceptuales y cognitivos (Riddoch y Humphreys, 1987; Ellis y Young, 1988; Riddoch et al., 1988; ver revisión de Manning, 1992) y ha sido Schacter (1990a) el primero que los ha relacionado con los fenómenos de memoria implícita. Aunque estos subsistemas se hallan descritos en diferentes sectores de la investigación neuropsicológica, Schacter se ha centrado en los hallazgos con pacientes disléxicos y agnósicos.

Una de las características básicas de estos subsistemas es que procesan y representan información sobre la forma y la estructura de las palabras, los objetos y otros tipos de estímulos, pero no procesan ni representan información semántica o asociativa sobre ellos (e. g. Ellis y Young, 1988; Riddoch et al., 1988). En consecuencia, el PRS se considera un sistema presemántico de representación perceptual.

En el dominio verbal, por ejemplo, varios estudios han demostrado un acceso intacto al conocimiento visual u ortográfico de las palabras junto a un acceso alterado al significado de tales palabras. Funnell (1983) describió a una paciente incapaz de establecer relaciones semánticas sobre palabras familiares que sí podía leer en voz alta; además, esta paciente no podía leer en voz alta no - palabras pronunciables. Funnell concluyó que la lectura que esta paciente hacía de las 
palabras familiares no se basaba en estrategias de conversión grafema-fonema. Sartori, Masterson y Job (1987) describieron un caso similar de un paciente que podía leer en voz alta palabras familiares pero que era incapaz de clasificar tales palabras en categorías semánticas. Al igual que la paciente de Funnell, este paciente tenía alterada su capacidad para leer no - palabras. Schwartz, Saffran y Marin (1980) estudiaron a una paciente que no podía clasificar palabras en categorías semánticas ni podía emparejarlas con sus dibujos correspondientes. Sin embargo, esta paciente podía leer correctamente en voz alta tanto palabras regulares como palabras irregulares (e. g. blood, climb). Si tenemos en cuenta que las palabras irregulares, a diferencia de las regulares, no pueden leerse en inglés sobre la base de conversiones grafema-fonema, su capacidad para leer palabras irregulares indica que la paciente tenía acceso a una representación almacenada de la forma visual de la palabra. Por tanto, este caso, al igual que los anteriores, puede considerarse como la demostración de una disociación entre la representación de la forma visual de una palabra y la representación de su significado.

Los abundantes datos sobre disociaciones entre forma visual y significado de las palabras han cimentado la idea de que la información sobre la forma visual de una palabra es representada por un sistema o un subsistema diferente del que representa la información semántica sobre esa palabra. Warrington y Shallice (1980) han llamado al sistema que preserva la forma el sistema de la forma visual de las palabras. Datos procedentes de investigaciones sobre neuroimágenes con la técnica PET (tomografía por emisión de positrones) han aportado evidencia convergente sobre la existencia de un sistema de la forma visual de las palabras (Petersen et al., 1988).

En el dominio no verbal, otros estudios neuropsicológicos sugieren la existencia de un sistema presemántico que representa la información sobre la forma y la estructura de los objetos visuales. La evidencia clave procede de estudios de pacientes con alguna forma de agnosia visual, que les incapacita para reconocer objetos familiares (ver revisiones de Humphreys y Riddoch, 1987, y Manning, 1992). Riddoch y Humphreys (1987) describen un paciente con un déficit severo de modalidad específica para nombrar y reconocer objetos presentados visualmente. Este paciente era incapaz de decir el nombre de un objeto si se le presentaba visualmente, pero no tenía dificultad para nombrarlo si se le hacía una descripción del mismo objeto auditivamente. Además, este paciente no podía responder a preguntas que exigían un acceso al conocimiento semántico de objetos visuales, ni sabía cómo emparejar dibujos de objetos con los nombres de sus categorías. Sin embargo, cuando las mismas tareas semánticas se le presentaban auditivamente, su rendimiento mejoraba considerablemente. Frente a esta incapacidad para acceder al conocimiento semántico sobre objetos visuales, este paciente tenía un rendimiento normal en tareas que requerían conocimiento sobre la estructura de los objetos. Por ejemplo, ejecutaba con normalidad una tarea de decisión de objetos en la que tenía que decidir si dibujos lineales representaban o no objetos reales.

Sobre la base de este y otros hallazgos (e. g. Sartori y Job, 1988) se ha argumentado que el conocimiento de la forma y la estructura de los objetos está representado en un sistema de base estructural distinto de un sistema semántico que representaría el conocimiento asociativo ofuncional de los mismos objetos (Warrington, 1982; Humphreys y Riddoch, 1987; Riddoch y Humphreys, 1987; Riddoch et al., 1988). Este sistema ha sido llamado sistema de descripción estructural (Riddoch et al., 1988). 
Schacter (1990a) considera al sistema de la forma visual y al sistema de descripción estructural subsistemas componentes del sistema de representación perceptual o PRS; el primero ejecuta sus funciones en el dominio verbal y el segundo, de un modo similar, las ejecuta en el dominio de los objetos. Teniendo en cuenta, por un lado, la evidencia de un sistema que procesa y representa la información sobre la forma visual de las palabras y que es independiente del sistema que procesa y representa la información semántica de tales palabras, y, por otro, demostraciones igualmente evidentes de la existencia de otro sistema que procesa y representa la información sobre la forma y la estructura de los objetos, independiente del sistema que representa el conocimiento asociativo y funcional sobre los mismo objetos, Schacter (1990a) se pregunta sobre las implicaciones de estos sistemas en la explicación de los efectos de priming y concluye que ambos sistemas juegan un papel muy significativo en tales efectos.

Apoyándose en estudios propios sobre el papel del priming en la identificación de palabras por parte de un «lector letra-a-letra» (Schacter et al., 1990), así como sobre el papel del priming en tareas de decisión de objetos (Schacter et al., 1990a, b; Schacter et al., 1991), Schacter ha obtenido datos consistentes acerca del crucial papel subyacente que juegan los sistemas de la forma de las palabras y de descripción estructural en los efectos de priming perceptual. Su conclusión básica es que los fenómenos de memoria implícita que requieren un procesamiento guiado por los datos (e. g., todos los tests implícitos no semánticos) reflejan la operación de subsistemas componentes del sistema de representación perceptual (PRS), cuya función es el procesamiento y la representación de la forma y la estructura en diferentes dominios del input. El PRS no debe considerarse, pues, implicado en todos los fenómenos de priming o de memoria implícita. Por tanto, los tests que requieren un procesamiento guiado conceptualmente (ver Masson, 1989; Roediger et al. , 1989; Blaxton, 1989) no implicarían al PRS, ya que dependen de la activación de formas de memoria episódica y/o semántica. Dicho de otra manera, el priming perceptual, reflejo de la actividad de PRS, se expresaría en pruebas implícitas guiadas por los datos, mientras que el priming conceptual, reflejo de los sistemas episódico y semántico, se expresaría en pruebas también implícitas pero guiadas conceptualmente. De acuerdo con ello, la propuesta de Schacter (1990a) permite hacer predicciones sobre la ocurrencia o no de disociaciones entre tests implícitos, especialmente entre los que implicarían al PRS y aquellos otros que involucrarían al sistema semántico (ver Blaxton, 1989), y todo ello no supondría -en contra de lo que opinan los teóricos de procesamiento- socabar las ideas de los proponentes de diferentes sistemas de memoria para explicar los efectos de priming.

La evidencia empírica y las propuestas teóricas aportadas por Schacter (1990a; Schacter et al., 1990a, b; Schacter et al., 1991), junto con todos los hechos empíricos sobre disociaciones presentados en este trabajo, han llevado a Tulving y Schacter (1990) a la conclusión de que priming (perceptual) es la expresión del sistema de representación perceptual o PRS que opera a un nivel presemántico. Esta hipótesis del PRS es considerada como la versión más elaborada de la hipótesis del «sistema QM sin huellas» (Hayman y Tulving, 1989a, b) y supone la culminación de las ideas expresadas hace ya un década (Tulving et al., 1982) sobre un sistema responsable de los efectos de priming que es independiente del sistema episódico y semántico pero que era «todavía poco conocido». Por tanto, el término PRS reemplaza al término «sistema QM».

Así pues, la evidencia revisada parece converger en que el sistema de repre- 
sentación perceptual vendría definido por las siguientes propiedades: 1) está involucrado en la identificación de objetos perceptuales, incluyendo las palabras; 2) sus computaciones neurales no dependen de forma crítica de las zonas cerebrales implicadas en las operaciones de la memoria episódica y semántica; 3) se desarrolla en las fases tempranas de la vida y se mantiene preservado diferencialmente en la vejez; 4) es relativamente inmune a los efectos de las drogas que afectan a otros sistemas de memoria; 5) sus operaciones se realizan al margen de la conciencia, por lo que sus productos no permiten al sujeto percatarse de los eventos y experiencias previas; 6) la información que representa está distribuida en representaciones múltiples de palabras y objetos concretos, y 7) el acceso a la información que contiene es hiperespecífico, probablemente porque no contiene, como otros sistemas de memoria, huellas focales o abstractas (Tulving y Schacter, 1990).

A partir de estas puntualizaciones, Tulving y Schacter (1990) concluyen que los efectos de priming o, más exactamente, el sistema de representación perceptual debe ser considerado como una nueva categoría de memoria implícita que no es ni procedimental ni semántica ni episódica (aunque tiene afinidades con la memoria procedimental y la memoria semántica), cuya función es mejorar la identificación de objetos perceptuales.

\section{Referencias}

Baddeley, A., y Whrrington, E. (1970). Amnesia and the distinction between long- and short-term memory. Journal of Verbal Learning and Verbal Bebavior, 9, 176-189.

BLAXTON, T. A. (1989). Investigating dissociations among memory measures: Support for a transfer-appropiate processing framework. Journal of Experimental Psychology: Learning, Memory and Cognition, 15, 657-668.

Butters, N.; Heindel, W. C., y Salmon, D. P. (1990). Dissociation of implicit memory in dementia: Neurological implications. Bulletin of the Psychonomic Society, 28, 359-366.

Carroll, M.; Byrne, B., y Kirsner, K. (1985). Autobiograhical memory and perceptual learning: A developmental study using picture recognition, naming latency, and perceptual identification. Memory and Cognition, 13, 273.279.

Cermak, L. S.; Talbot, N.; Chandler, K., y Wolbarst, L. R. (1985). The perceptual priming phenomenon in amnesia. Neuropsychology, 23, 615-622.

COFEr, CH. N. (1967). Conditions for the use of verbal associations. Psychological Bulletin, $68,1-12$

Comen, N. J. (1984). Preserved learning capacity in amnesia: Evidence for multiple memory systems. En L. R. Squire y N. Butters (Eds.), Neuropsychology of memory. Nueva York: Guilford Press.

Cohen, N. J., y Squire, L. R. (1980). Preserved learning and retention of pattern-analyzing skill in amnesia: Dissociation «knowing how» and «knowing taht». Science, 210, 207-209.

Corkin, S. (1968). Acquisition of motor skill after bilateral medial temporal-lobe excision. Neuropsychologia, 6, 255-266.

CraIK, F. I. M., y Tulving, E. (1975). Depth of processing and the retention of words in episodic memory. Joumal of Experimental Psychology: General, 104, 268-294.

Chander, C. (1983). Does retrieval strategy determine the relation between episodic recognition and semantic priming? Master's thesis. Universidad de Toronto, Toronto, Canadá.

Diamond, R., y Rozin, P. (1984). Activation of existing memories in anterograde amnesia. Journal of Abnormal Psychology, 93, 98-105.

Durgunoglu, A., y Roediger, H. L. (1987). Test differences in accessing bilingual memory. Journal of Memory and Language, 26, 377-391.

Durso, F. T., y Johnson, M. K. (1979). Facilitation in naming and categorizing repeated pictures and words. Joumal of Experimental Psychology: Human Learning and Memory, 5, 449.459.

EICH, E. (1984). Memory for unattended events: Remembering with and without awareness. Memory and Cognition, 12, 105-111. 
Ellis, A., y Young, A. (1988). Human cognitive neuropsychology. Londres: LEA.

Feustel, T. C.; Shiffrin, R. M., y Salasoo, A. (1983). Episodic and lexical contributions to the repetition effect in word identification. Joumal of Experimental Psychology: General, 112, 309-346.

Fodor, J. A. (1983). The modularity of mind. Cambridge, MA: Bradford.

Froufe, M. (1991). Memoria y conciencia. En J. M. Ruiz-Vargas (Dir), Psicología de la memoria. Madrid: Alianza.

FunNell, E. (1983). Phonological processes in reading: New evidence from acquired dyslexia. British Joumal of Psychology, 74, 159-180.

Ghoneim, M. M.; Block, R. I.; Ping, S. T. S.; Ali, M. A., y Hoffman, J. G. (1990). Learning without recall during general anaesthesia. En B. Bonke, W. Fitch y K. Millar (Eds.) Memory and awareness in anaesthesia. Amsterdam: Swets \& Zeitlinger.

GRAF, P. (1990). Life-span changes in implicit and explicit memory. Bulletin of The Psychonomic Society, 28, 353-358.

GraF, P., y Mandler, G. (1984). Activation makes words more accesible, but not necessarily more retrievable. Journal of Verbal Learning and Verbal Bebavior, 23, 553-568.

Graf, P.; MANDler, G., y Haden, P. E. (1982). Simulating amnesic symptoms in normals. Science, 218, 1.243-1.244.

Graf, P., y SCHACTER, D. L. (1985). Implicit and explicit memory for new associations in normal and amnesics subjects. Joumal of Experimental Psychology: Learning, Memory and Cog. nition, 11, 386-396.

GrAF, P., y SCHACTER, D. L. (1989). Unitization and grouping mediate dissociations in memory for new associations. Journal of Experimental Psycholgy: Learning, Memory and Cog. nition, $15,930-940$.

Graf, P.; Shimamura, A. P., y Souire, L. R. (1985). Priming acrosss modalities and priming across category levels: Extending the domain of preserved function in amnesia. Joumal of Experimental Psychology: Learning, Memory and Cognition, 11, 386-396.

Graf, P.; Squire, L. R., y Mandler, G. (1984). The information that amnesic patients do not forget. Joumal of Experimental Psychology: Leaming Memory and Cognition, 10, 164-178.

Greenbaum, J. L., y Graf, P. (1989). Preschool period development of implicit and explicit remembering. Bulletin of the Psychonomic Society, 27, 417-420.

Hatroudi, S.; Parker, E. S.; DELisi, L. E.; Wyatt, R. J., y Mutter, S. A. (1984). Intact retention in acute alcohol amnesia. Joumal of Experimental Psycholgy: Learming, Memory and Cognition, 10, 156-163.

Hayes.Roth, B. (1977). Evolution of congnitive structures and processes. Psychological Revivew, $84,260-278$.

Hayman, C. A. G., y Tulving, E. (1989a). Contingent dissociation between recognition and fragment completion: The method of triangulation. Journal of Experimental Psychology: Learning, Memory and Cognition, 15, 228-240.

Hayman, C. A. G., y Tulving, E. (1989b). Is priming in fragment completion based on a «traceless» memory system? Journal of Experimental Psychology: Learning, Memory and Cognition, 15, 941-956.

Heindel, W. C.; Salmon, D. P.; Shults, C.; Walicke, P., y Butters, N. (1989). Neuropsychological evidence for multiple implicit memory systems: A comparison of Alzheimer's, Huntington's and Parkinson's disease. The Journal of Neuroscience, 9, 582-587.

Hintzman, D. L. (1990). Human learning and memory: Connections and dissociations. Annual Review of Psychology, 41, 109-139.

Howard, D. V. (1988). Aging and memory activation: The priming of semantic and episodic memories. En L. L. Light y D. M. Burke (Eds.), Language, memory, and aging. Cambridge, MA: Cambridge University Press.

Humphreys, G. W., y RidDoch, M. J. (1987). Visual object processing: A cognitive neuropsychological approach. Londres: LEA.

IDZIKowsKI, C. (1988). The effects of drugs on human memory. En M. M. Gruneberg, P. E. Morris y R. N. Sykes (Eds.), Practical aspects of memory. Vol. 2. Chichester: Wiley.

JACOBY, L. L. (1983). Remembering the data: Analyzing interactive processes in reading. Journal of Verbal Learning and Verbal Bebavior, 22, 485-508.

JACOBY, L. L. (1988). Memory observed and memory unobserved. En U. Neisser y E. Winograd (Eds.), Remembering reconsidered. Cambridge: Cambridge University Press.

JACOBY, L. L., Y DALLAS, M. (1981). On the relationship between autobiographical memory and perceptual learning. Joumal of Experimental Psychology: General, 110, 306-340.

JACOBY, L. L., Y WITHERSPOON, D. (1982). Remembering without awareness. Canadian Journal of Psychology, 36, 300-324

Java, R. I., y Gardiner, J. M. (1991). Priming and aging: Further evidence of preserved memory function. American Joumal of Psychology, 104, 89-100. 
Johnson, M. K. (1983). A multiple-entry, modular memory system. En G. H. Bower (Ed.), The psychology of learning and motivation. Vol. 17. Nueva York: Academic Press.

Johnson, M. K., y Hasher, L. (1987). Human learning and memory. Annual Review of Psychology, 38, 631-668.

Kirsner, K.; Milech, D., y Standen, P. (1983). Common and modality-specific processes in the mental lexicon. Memory and Cognition, 11, 621-630.

Kirsner, K.; Milech, D., y Stumpfl, V. (1986). Word and picture identification: Is representational parsimony possible? Memory and Cognition, 14, 398-408.

Kolers, P. A., Y Roediger, H. L. (1984). Procedures of mind. Joumal of Verbal Learning and Verbal Behavior, 23, 425-449.

Kомaтsu, S. I., y Oнта, N. (1984). Priming effects in word-fragment completion for shortand long-term retention intervals. Japanese Psychological Research, 26, 194-200.

Kopelman, M. D., y CORN, T. H. (1988). Cholinergic «blocade» as a model for cholinergic depletion. Brain, 111, 1.079-1.110.

LigHT, L. L. (1988). Preserved implicit memory in old age. En M. M. Gruneberg, P. Morris y R. N. Sykes (Eds.), Practical aspects of memory. Vol 2. Chichester: Wiley.

Light, L. L. (1991). Memory and aging: Four hipothesis in search of data. Annual Review of Psychology, 42, 333-376.

LIGHT, L. L., Y SINGH, A. (1987). Implicit and explicit memory in young and older adults. Journal of Experimental Psychology: Learning, Memory and Cognition, 13, 531-541.

LIGHT, L. L.; SINGH, A., y CAPPS, J. L. (1986). Dossociation of memory and awareness in young and older adults. Journal of Clinical and Experimental Neuropsychology, 8, 62.74.

MANDlfr, G. (1980). Recognizing: The judgment of previous occurrence. Psychological Review, 87, 252-271.

MANDLER, G. (1989). Memory: Conscious and unconscious. En P. R. Solomon, G. R. Goethals, C. M. Kelley y B. R. Stephens (Eds.). Memory: Interdisciplinary approaches. Nueva York: Springer.

Mandler, G.; Graf, P., y Kraft, D. (1986). Activation and elaboration effects in recognition and word priming. Quarterly Joumal of Experimental Psychology, 38A, 645-662.

MANNING, L. (1992). Introducción a la neuropsicología clásica y cognitiva del lenguaje. Madrid: Trotta.

MASSON, M. E. (1989). Fluent reprocessing as an implicit expression of memory for experience. En S. Lweandowsky, J. Dunn y K. Kirsner (Eds.), Implicit memory: Theoretical issues. Hillsdale, N. J.: LEA.

MaYes, A. R. (1988). What functional deficits underlie organic amnesia? En M. M. Gruneberg, P. E. Morris y R. N. Sykes (Eds.), Practical aspects of memory. Vol. 2. Chischester: Wiley.

MCANDREws, M. P.; GlISKY, E. L., y SCHACTER, D. L. (1987). When priming persists: longlasting implicit memory for a single episode in amnesic patients. Neuropsychologia, 25, 497-506.

Merikle, P. M., y Reingold, E. M. (1991). Comparing direct (explicit) and indirect (implicit) measures to study unconscious memory. Joumal of Experimental Psychology: Learning, Memory and Cognition, 17, 224.233.

Mishrin, M.; Malamut, B., y Bachevalier, J. (1984). Memories and habits: Two neural systems. En G. Lynch, J. L. McGaugh y N. Weinberger (Eds.), Neurobiology of learning and memory. Nueva York: Guilford Press.

MitcheLl, D. B., y BROWN, A. S. (1988). Persistent repetition priming in picture naming and its dissociation from recognition memory. Joumal of Experimental Psychology: Learning, Memory and Cognition, 14, 213.222.

MORRIS, C. D.; BranSFord, J. D., y FranKs, J. J. (1977). Levels of processing versus transfer appropiate processing. Journal of Verbal Learnintg and Verbal Bebavior, 16, 519-533.

MORTON, J. (1969). Interaction of information in word recognition. Psychological Review, 76, $165-178$.

MorTon, J. (1979). Facilitation in word recognition: Experiments causing change in the logogen models. En P. A. Kolers, M. E. Wrolstad y H. Bouma (Eds.), Processing of visible language. Nueva York: Plenum.

Morton, J., y Patterson, K. E. (1980). A new attempt at an interpretatión, or an attempt at a new interpretation. En M. Coltheart, K. E. Patterson y J. C. Marsshall (Eds.), Deep dyslexia. Londres: Routledge and Kegan Paul.

Moscovitch, M. (1982). Multiple dissociations of function in amnesia. En L. S. Cermak (Ed.), Human memory and amnesia. Hillsdale, N. J.: LEA.

Moscovitch, M.; WinOCUR, G., Y MCLACHLAN, D. (1986). Memory as assessed by recognition and reading time in normal and memory-impaired people with Alzheimer's disease and other neurological disorders. Joumal of Experimental Psychology: General, 115, 331-347. 
Neisser, U. (1978). Memory: What are the important questions? En M. M. Gruneberg, P. E. Morris y R. N. Sykes (Eds.), Practical aspects of memory. Londres: Academic Press.

Nissen, M. J.; Knopman, D., y Schacter, D. L. (1987). Neurochemical dissociation of memory systems. Neurology, 37, 789-794.

Parkin, A. J., y Streete, S. (1988). Implicit and explicit memory in young children and adults. British Journal of Psychology, 79, 361-369.

Petersen, S. E.; Fox, P. T.; Posner, M. I.; Mintum, M., y Raichle, M. E. (1988). Positron emission tomographic studies of the cortical anatomy of single-word processing. Nature, 331, 585-589.

Polster, M. R.; Gray, P. A.; McCarthy, R. A. y PARK, G. R. (1990). Implicit and explicit memory after intra-muscular midazolam. En B. Bonke, W. Fitch y K. Millar (Eds.), Memory and awareness in anaesthesia. Amsterdam: Swets \& Zeitlinger.

Richardson.Klavehn, A., y Bjork, R.A. (1988). Measures of memory. Annual Review of Psychology, 39, 475-543.

RidDoch, M. J., y Humphreys, G. W. (1987). Visual object processing in optic aphasia: A case of semantic access agnosia. Cognitive Neuropsychology, 4, 131-186.

Riddoch, M. J.; Humphreys, G. W.; Coltheart, M., y Funnell, E. (1988). Semantic system or systems? Neuropsychogical evidence re-examined. Cognitive Neuropsycbology, 5, 3-26.

Roediger, H. L. (1984). Does currente vidence from dissociation experiments favor the episodic/semantic distinction? The Behavioral and Brain Sciences, 7, 252-254.

Roediger, H. L. (1990a). Implicit memory: Retention without remembering. American Psychologist, 45, 1.043-1.056.

Roediger, H. L.(1990b). Implicit memory: A commentary. Bulletin of the Psychonomic So ciety, 28, 373-380.

Roediger, H. L., y Blaxton, T. A. (1987a). Retrieval modes produce dissociations in memory for surface information. En D. S. Gorfein y R. R. Hoffman (Eds.), Memory and learning: The Ebbinghaus Centennial Conference. Hillsdale, N. J.: LEA.

Roediger, H. L., y Blaxton, T. A. (1987b). Effects of varying modality, surface features, and retention interval on priming in word-fragment completion. Memory and Cognition, $15,379-388$

Roediger, H. L.; Rajaram, S., y Srinivas, K. (1990). Specifying criteria for postulating memory systems. Annals of the New York Academy of Sciences, 608, 572-595.

Roediger, H. L., y WELdON, M. S. (1987). Reserving the picture superiority effect. En M. A. McDaniel y M. Pressley (Eds.), Imagery and related mnemonic processes. Nueva York: Springer.

Roediger, H. L.; Weldon, M. S., y Challis, B. H. (1989). Explaining dissociations between implicit and explicit measures of retention: A processing account. En H. L. Roediger y F. I. M. Craik (Eds.), Varieties of memory and consciousness: Essays in Honour of Endel Tulving. Hillsdale, N. J.: LEA.

Roorda.Hrdlicková, V.; Wolters, G.; Bonke, B., y Phaf, R. H. (1990). Unconscious perception during general anaesthesia, demonstrated by and implicit memory task. En B. Bonke, W. Fitch y K. Millar (Eds.), Memory and awareness in anaestbesia. Amsterdam: Swets \& Zeitlinger.

Salasoo, A.; Shiffrin, R. M., y Feustel, T. C. (1985). Building permament memory codes: Codification and repetition effects in word identification. Joumal of Experimental Psychology: General, 114, 50-77.

SARTORI, G., Y JOB, R. (1988). The oyster with four legs: A neuropsychological study on the interaction of visual and semantic information. Cognitive Neuropsychology, 5, 105-132.

Sartori, G.; Masterson, J., y Job, R. (1987). Direct-route reading and the locus of lexical decision. En M. Coltheart, G. Sartori y R. Job (Eds.), The cognitive neuropsychology of language. Londres: LEA.

Scarborough, D. L.; Gerard, L., y Cortese, C. (1979). Accessing lexical memory: Transfer of word repetition effects across task and modality. Memory and Cognition, 7, 3-12.

Schacter, D. L. (1985). Priming of old and new knowledge in amnesic patients and normal subjects. Annals of the New York Academy of Sciences, 444, 41-53.

SCHACTER, D. L. (1987). Implicit memory: History and current status. Joumal of Experimental Psychology: Learning, Memory and Cognition, 13, 501-518.

SCHACTER, D. L. (1989). On the relation between memory and consciousness: Dissociable interactions and conscious experience. En H. L. Roediger y F. I. M. Craik (Eds.), Varieties of memory and consciousness: Essays in Honour of Endel Tulving. Hillsdale, N. J.: LEA.

SCHACTER, D. L. (1990a). Perceptual representation systems and implicit memory: Toward a resolution of the multiple memory systems debate. Annals of the New Yord Academy of Sciences, 608, 543-571. 


\section{5}

SCHACTER, D. L. (1990b). Introduction to «Implicit memory: Multiple perspectives». Bulletin of the Psychonomic Society, 28, 338-340.

SCHACTER, D. L. (1990c). Toward a cognitive neuropsychology of awareness: Implicit knowledge and anosognosia. Journal of Clinical and Experimental Neuropsycbology, 12, 155-178.

SCHACTER, D. L.; BOwERS, J., y BOOKER, J. (1989). Intention, awareness, and implicit memory: The retrieval intentionality criterion. En S. Lewandowsky, J. Dunn y K. Kirsner (Eds.), Implicit memory: Theoretical issues. Hillsdale, N. J.: LEA.

Schacter, D. L.; Cooper, L. A., y Delaney, S. M. (1990a). Implicit memory for unfamiliar objects depends on access to structural descriptions. Journal of Experimental Psychology: General, 119, 5-24.

Schacter, D. L.; Cooper, L. A., y Delaney, S. M. (1990b). Implicit memory for visual objects and the structural description system. Bulletin of the Psychonomic Society, 28, 367-372.

Schacter, D. L.; Cooper, L. A.; Delaney, S. M.; Peterson, M. A., y Tharan, M. (1991). Implicita memory for possible and impossible objects: Constraints on the construction of structural descriptions. Joumal of Experimental Psychology: Leaming, Memory and Cognition, 17, 3-19.

Schanter, D. L., Delaney, S. y Merikle, E. (1990). Priming of non-verbal information and the nature of implicit memory. En G. H. Bower (Ed.), The Psychology of Learning and Motivation. Vol. 26. Nueva York: Academic Press.

SChaCter, D. L., y Graf, P. (1986a). Effects of elaborative processing on implicit and explicit memory for new associations. Journal of Experimental Psychology: Learning, Memory and Cognition, 12, 432-444.

Schacter, D. L., y Graf, P. (1986b). Preserved learning in amnesic patients: Perspectives from research on direct priming. Joumal of Clinical and Experimental Neuropsycbology, 8, 727.743

Schacter, D. L., y Graf, P. (1989). Modality specificity of implicit memory for new associations. Journal of Experimental Psychology: Learning, Memory and Cognition, 15, 3-12.

Schacter, D. L.; McAndrews, M. P., y Moscovitch, M. (1988). Access to consciousness: Dissociations between implicit and explicit knowledge in neuropsychological syndromes. En L. Weiskrantz (Ed.), Thought without language. Oxford: Clarendon Press.

SChacter, D. L., y McGlynn (1989). Implicit memory: Effects of elaboration depend on unitization. American Journal of Psychology, 102, 452-481.

SChacter, D. L., y Moscovitch, M. (1984). Infants, anmesics, and dissociable memory systems. En M. Moscovitch (Ed.), Infant memory. Nueva York: Plenum.

Schacter, D. L.; Rapscak, S.; Rubens, A.; Tharan, M., y Laguna, J. (1990). Priming effects in a letter-by-letter reader depend upon access to the word form system. Neuropsychologia.

Schacter, D. L., y Tulving, E. (1982). Memory, amnesia, and the episodic/semantic distinction. En R. L. Isaacson y N. E. Spear (Eds.), The expression of knowledge. Nueva York: Plenum.

Schwartz, M. F.; Saffran, E. M., y Marin, O. S. M. (1980). Fractionating the reading process in dementia: Evidence for word-specific print-to-sound associations. En M. Coltheart, K. Patterson y J. C. Marshall (Eds.), Deep dyslexia. Londres: Routledge and Kegan Paul.

Sherry, D. F., y Schacter, D. L. (1987). The evolution of multiple memory suystems. Psychological Review, 94, 439-454.

Shimamura, A. P. (1985). Problems with the finding of stochastic independence as evidence for multiple memory systems. Bulletin of the Psychonomic Society, 23, 506-508.

Shimamura, A. P. (1986). Priming effects in amnesia: Evidence for a dissociable memory function. Quarterly Journal of Experimental Psychology, 38A, 619-644.

Shimamura, A. P. (1989). Disorders of memory: The cognitive science perspective. En F. Boller y J. Grafman (Eds.), Handbook of Neurospychology. Vol. 3. Amsterdam: Elsevier.

Shimamura, A. P., y Souire, L. R. (1984). Paired-associate learning and priming effects in amnesia: A neuropsychological approach. Journal of Experimental Psychology: General, 113, 556-570.

Shimamura, A. P., y Souire, L. R. (1989). Impaired priming of new associations in amnesia. Journal of Experimental Psychology: Learning, Memory and Cognition, 15, 721-728.

Sloman, S. A.; Hayman, C. A.; Ohta, N.; Law, J., y Tulving, E. (1988). Forgetting in primed fragment completion. Joumal of Experimental Psychology: Learning, Memory and Cognition, 14, 223-239.

Sovire, L. R. (1986). Mechanisms of memory. Science, 232, 1.612-1.619.

Squire, L. R. (1987). Memory and brain. Nueva York: Oxford University Press.

Squire, L. R., y Cohen, N. J. (1984). Human memory and amnesia. En G. Lynch, J. L. McGaugh y N. Weinberger (Eds.), Neurobiology of learning and memory. Nueva York: Guilford Press. 


\section{6}

Squire, L. R., y Davis, H. P. (1981). The pharmacology of memory: A neurobiological perspective. Annual Review of Pharmacology and Toxicology, 21, 323-356.

Souire, L. R.; ShimamuRa, A. P., y Graf, P. (1985). Independence of recognition memory and priming effects: A neuropsychological analysis. Journal of Experimental Psychology: Learning, Memory and Cognition, 11, 37-44.

Squire, L. R.; Shimamura, A. P., y Graf, P. (1987). Srengh and duration of priming effects in normal subjects and amnesic patients. Neuropsychologia, 25, 195-210.

Tulving, E. (1972). Episodic and semantic memory. En E. Tulving y W. Donaldson (Eds.), Organization of memory. Nueva York: Academic Press.

Tulving, E. (1983). Elements of episodic memory. Nueva York: Oxford University Press.

Tulving, E. (1985). How many memory systems are there? American Psychologist, 40, 385-398.

Tuiving, E. (1987). Multiple memory systems and consciousness. Human Neurobiology, 6, 67-80.

Tulving, E., y Schacter, D. L. (1990). Priming and human memory systems. Science, 247, 301-306.

Tulving, E.; Schacter, D. L., y Stark, H. A. (1982). Priming effects in word-fragment completion are independent of recognition memory. Joumal of Experimental Psychology: Learning, Memory and Cognition, 8, 336-342.

Tulving, E., Y THOMson, D. M. (1973). Encoding specificity and retrieval processes in episodic memory. Psychological Review, 80, 352-373.

WarRINGton, E. (1982). Neuropsychological studies of object recognition. Pbilosopbical Transactions of the Royal Society, LondonB298, 15-33.

Warrington, E., y Shallice, T. (1980). Word-form dyslexia. Brain, 103, 99-112.

WarRington, E., y WeISKRANTZ, L. (1968). New method for testing long-term retention with special reference to amnesic patients. Nature, 217, 972-974.

WarRington, E., y Weiskrantz, L. (1970). Amnesic syndrome: Consolidation or retrieval? Nature, 228, 629-630.

Warrington, E., Y Weiskrantz, L. (1982). Amnesia: A disconnection syndrome? Neuropsychology, 20, 233-248.

WeISKRANTZ, L. (1987). Neuroanatomy of memory and amnesia: A case for multiple memory systems. Human Neurobiology, 6, 93-105.

WeISKRANTZ, L. (1989). Remembering dissociations. En H. L. Roediger y F. I. M. Craik (Eds.), Varieties of memory and consciousness: Essays in Honour of Endel Tulving. Hillsdale, N. J.: LEA.

WELDON, M. S., Y RoEDIGER, H. L. (1987). Altering retrieval demands reverses in the picture superiority effect. Memory and Cognition, 15, 269-280.

WITHERSPOON, D., y Moscovitch, M. (1989): Stochastic independence between two implicit memory tasks. Joumal of Experimental Psychology: Learning, Memory and Cognition, 15, $22-30$. 